\title{
Endocannabinoid signaling in hypothalamic circuits regulates arousal from general anesthesia in mice
}

\author{
Haixing Zhong, ${ }^{1,2,3}$ Li Tong, ${ }^{1,4}$ Ning Gu, ${ }^{1,2}$ Fang Gao, ${ }^{1,2,5}$ Yacheng Lu, ${ }^{6}$ Rou-gang Xie, ${ }^{3,5}$ Jingjing Liu, ${ }^{3}$ Xin Li, ${ }^{1,2,3}$ Richard Bergeron, ${ }^{2}$ \\ Lisa E. Pomeranz, ${ }^{7}$ Ken Mackie, ${ }^{8}$ Feng Wang, ${ }^{1,2,3}$ Chun-Xia Luo, ${ }^{1}$ Yan Ren, ${ }^{1}$ Sheng-Xi Wu, ${ }^{5}$ Zhongcong Xie, ${ }^{9}$ Lin Xu, ${ }^{10}$ Jinlian Li, ${ }^{6}$ \\ Hailong Dong, ${ }^{3}$ Lize Xiong, ${ }^{3}$ and Xia Zhang ${ }^{1,2,3}$ \\ ${ }^{1}$ Institute of Mental Health Research at the Royal, and ${ }^{2}$ Departments of Psychiatry and Cellular \& Molecular Medicine, University of Ottawa, Ottawa, Ontario, Canada. ${ }^{3}$ Department of Anesthesiology and \\ Perioperative Medicine, Xijing Hospital, Fourth Military Medical University, Xi'an, China. ${ }^{4}$ Anesthesia and Operation Center, Chinese PLA General Hospital, Beijing, China. ${ }^{5}$ Department of Neurobiology, and \\ ${ }^{6}$ Department of Anatomy, Histology, and Embryology, Fourth Military Medical University, Xi'an, China. 'Laboratory of Molecular Genetics, Rockefeller University, New York, New York, USA. ${ }^{8}$ Department of \\ Psychological and Brain Sciences, Indiana University, Bloomington, Indiana, USA. ${ }^{9}$ Department of Anesthesia, Critical Care and Pain Medicine, Massachusetts General Hospital, Harvard Medical School, \\ Charlestown, Massachusetts, USA. ${ }^{10}$ Key Lab of Animal Models and Human Disease Mechanisms, Kunming Institute of Zoology, Chinese Academy of Science, Kunming, China.
}

\begin{abstract}
Consciousness can be defined by two major attributes: awareness of environment and self, and arousal, which reflects the level of awareness. The return of arousal after general anesthesia presents an experimental tool for probing the neural mechanisms that control consciousness. Here we have identified that systemic or intracerebral injection of the cannabinoid $\mathrm{CB}_{1}$ receptor ( $\left.\mathrm{CB}_{1} \mathrm{R}\right)$ antagonist $\mathrm{AM} 281$ into the dorsomedial nucleus of the hypothalamus (DMH) - but not the adjacent perifornical area (Pef) or the ventrolateral preoptic nucleus of the hypothalamus (VLPO) - accelerates arousal in mice recovering from general anesthesia. Anesthetics selectively activated endocannabinoid (eCB) signaling at DMH glutamatergic but not CABAergic synapses, leading to suppression of both glutamatergic DMH-Pef and CABAergic DMH-VLPO projections. Deletion of CB1R from widespread cerebral cortical or prefrontal cortical (PFC) glutamatergic neurons, including those innervating the DMH, mimicked the arousal-accelerating effects of AM281. In contrast, CB1R deletion from brain CABAergic neurons or hypothalamic glutamatergic neurons did not affect recovery time from anesthesia. Inactivation of PFC-DMH, DMHVLPO, or DMH-Pef projections blocked AM281-accelerated arousal, whereas activation of these projections mimicked the effects of AM281. We propose that decreased eCB signaling at glutamatergic terminals of the PFC-DMH projection accelerates arousal from general anesthesia through enhancement of the excitatory DMH-Pef projection, the inhibitory DMH-VLPO projection, or both.
\end{abstract}

\section{Introduction}

Consciousness is an essential human attribute (1), but the neural mechanism controlling consciousness remains unknown $(2,3)$. Consciousness consists of two major components: arousal (i.e., the level of consciousness) and awareness of the environment and self (i.e., the content of consciousness). Arousal after cessation of general anesthesia provides an experimental model to investigate the neural mechanism that controls consciousness (3-6), as yearly more than 312 million patients are placed under general anesthesia worldwide (7). Although the exact mechanism underlying arousal from anesthesia is unknown, ample evidence suggests a remarkable similarity between anesthesia-induced unconsciousness and deep sleep $(1,3,4,6,8)$. The hypothalamus is a key brain region for regulation of sleep and wakefulness (9-13). Specifically, the dorsomedial nucleus of the hypothalamus (DMH) conveys and reorganizes circadian rhythms of sleep and wakefulness from the suprachiasmatic nucleus to both the wake-promoting perifornical

Authorship note: H. Zhong, L. Tong, N. Gu, and F. Gao contributed equally to this work Conflict of interest: The authors have declared that no conflict of interest exists.

Submitted: October 3, 2016; Accepted: March 2, 2017.

Reference information: J Clin Invest. 2017;127(6):2295-2309.

https://doi.org/10.1172/JCI91038 area (Pef) and the sleep-promoting ventrolateral preoptic nucleus (VLPO) (9-13). The DMH receives both GABAergic innervation from the suprachiasmatic nucleus (SCN) (9-13) and glutamatergic innervation from the prelimbic and infralimbic areas of the prefrontal cortex (PFC) (14) and then sends GABAergic axons innervating VLPO GABA-containing neurons and glutamatergic axons innervating Pef orexin-containing neurons (Supplemental Figure 1; supplemental material available online with this article; https:// doi.org/10.1172/JCI91038DS1) (9-13). However, it is unknown whether and how glutamatergic PFC-DMH, GABAergic SCN$\mathrm{DMH}$, glutamatergic DMH-Pef, and GABAergic DMH-VLPO projections modulate arousal after general anesthesia.

Two well-characterized endogenous cannabinoids (endocannabinoids [eCBs]) are 2-arachidonoylglycerol and anandamide (or $\mathrm{N}$-arachidonoylethanolamine), which are mainly hydrolyzed by monoacylglycerol lipase (MAGL) and fatty acid amide hydrolase (FAAH), respectively (15). It is well established that $\mathrm{eCB}$ activation of presynaptic cannabinoid $\mathrm{CB}_{1}$ receptor $\left(\mathrm{CB}_{1} \mathrm{R}\right)$, the most abundant $G$ protein-coupled receptor in the brain (16), inhibits release of glutamate and GABA from glutamatergic and GABAergic axon terminals, respectively $(17,18)$. However, it is not known whether and how eCB signaling at synapses of PFC-DMH, SCN-DMH, DMH-Pef, and DMH-VLPO projections participates in the pro- 

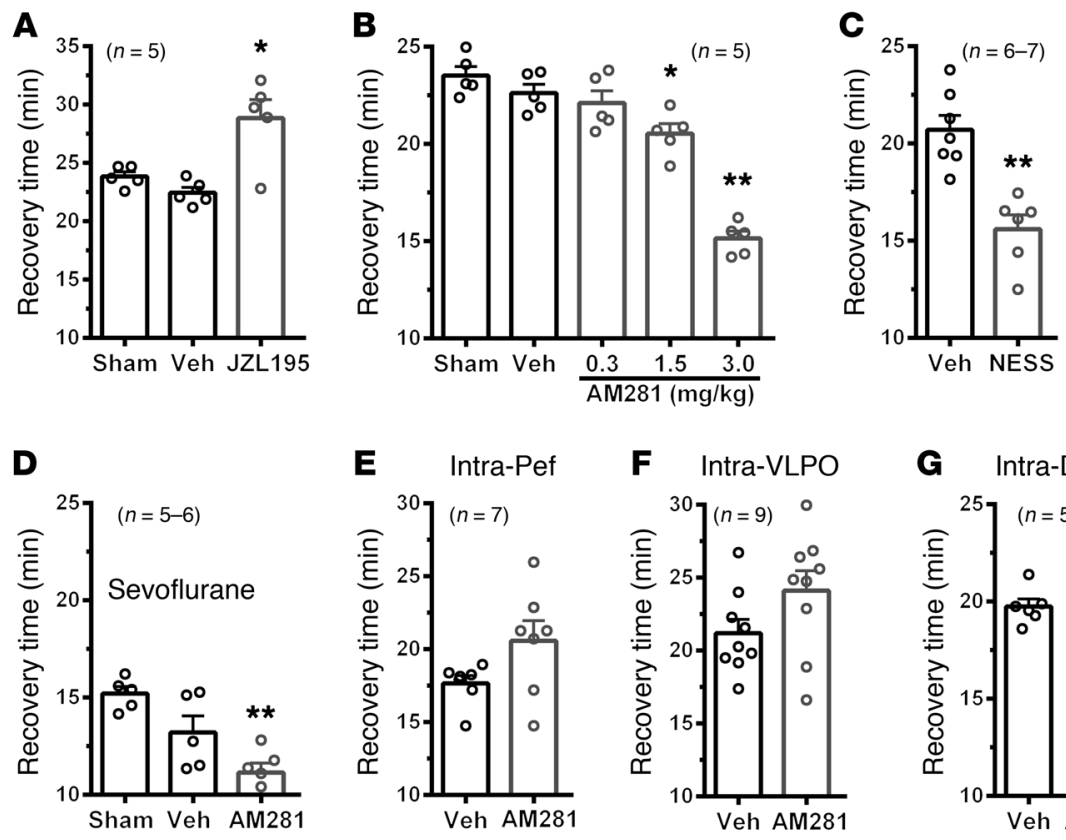

cess of arousal after general anesthesia. Loss of wakefulness and arousal from anesthesia in rodents can be quantified as the time to loss or resumption of righting responses (LORR or RORR), respectively, following application or cessation of general anesthesia. A recent study showed that $\mathrm{CB}_{1} \mathrm{R}$ blockade suppressed LORR following administration of the anesthetic propofol (19), indicating that anesthetic-increased eCB signaling enhances anesthetic induction of unconsciousness. We therefore hypothesized that decreased eCB signaling at PFC-DMH, SCN-DMH, DMH-Pef, and/or DMH-VLPO projections may participate in arousal after general anesthesia.

In the present study, we critically examine this hypothesis by employing behavioral, electrophysiological, electron microscopic, molecular biological, conditional mutagenetic, and chemogenetic strategies. General anesthesia was induced by administration of isoflurane or sevoflurane, two volatile anesthetics commonly used in clinical practice. Our findings suggest that eCB signaling at axon terminals of glutamatergic $\mathrm{PFC}-\mathrm{DMH}$ projection, but not GABAergic SCN-DMH projection, and subsequent involvement of both glutamatergic DMH-Pef and GABAergic DMH-VLPO projections participate in arousal after general anesthesia.

\section{Results}

eCB modulation of arousal from anesthesia through the DMH. Anesthesia was delivered to mice in a standard anesthetic chamber (Supplemental Figure 2) (20). We quantified RORR time (i.e., emergence or recovery time) following a 30-minute exposure to isoflurane or sevoflurane anesthesia, with pharmacological interventions performed 15 minutes before the end of anesthesia. We first quantified recovery time in association with a continuous EEG recording following isoflurane anesthesia. When rats showed RORR, their EEG spectrum displayed a sudden change from a generally high-power to low-power state (Supplemental Figure 3), clearly suggesting the reliability of measuring RORR time for the quantification of brain arousal after cessation of general anesthesia.
Figure 1. eCB modulation of arousal from anesthesia. (A and $\mathbf{B}$ ) Relative to sham anesthesia (Sham), JZL195 (A: $20 \mathrm{mg} / \mathrm{kg}$, i.p.) and AM281 (B: $0.3,1.5,3.0 \mathrm{mg} / \mathrm{kg}$, i.p.) - but not vehicle (Veh) - respectively prolong and shorten recovery time after isoflurane anesthesia. (C) Relative to Veh, NESS0327 (NESS, $0.3 \mathrm{mg} / \mathrm{kg}$, i.p.) significantly shortens recovery time after isoflurane anesthesia. (D) Relative to sham treatment, AM281 (3 $\mathrm{mg} / \mathrm{kg}$, i.p.) but not Veh significantly shortens recovery time after sevoflurane anesthesia. (E and F) Neither intra-Pef (E) nor intra-VLPO microinjection (F) of AM281 (0.1 $\mu \mathrm{g} / 0.3 \mu \mathrm{l} / \mathrm{side})$ significantly affects recovery time $(\mathrm{E}: P=0.0723 ; \mathbf{F}: P=0.231$ )

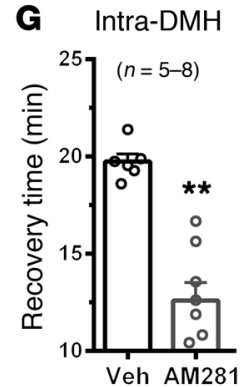
after isoflurane anesthesia. (G) Bilateral intraDMH microinjection of AM281 ( $0.1 \mu \mathrm{g} / 0.3 \mu \mathrm{l} / \mathrm{side})$ significantly shortens recovery time after isoflurane anesthesia. All summary graphs show mean $\pm \mathrm{SEM} ; n$, number of rats in each group. ${ }^{*} P<0.05$ and ${ }^{* *} P<0.01$ vs. sham or Veh, Tukey's post-hoc test after 1-way ANOVA (A: $F_{2,12}=11.63, P<0.05$; B: $\left.F_{4,20}=46.17, P<0.01 ; \mathbf{D}: F_{2,13}=12.13, P<0.01\right)$ or Student's $t$ test (C and $\mathbf{E}-\mathbf{G})$.

To examine whether increased eCB signaling modulates arousal after anesthesia, we injected JZL195 (20 mg/kg, i.p.), an inhibitor of both MAGL and FAAH (15). JZL195 significantly prolonged recovery time after isoflurane anesthesia (Figure 1A). We reasoned that if this longer recovery time resulted from increased eCB signaling, blocking eCB signaling should shorten recovery time. Indeed, an i.p. injection of the $\mathrm{CB}_{1} \mathrm{R}$ antagonist $\mathrm{AM} 281$ shortened recovery time after isoflurane anesthesia in a dosedependent manner, with the maximal effect produced by $3 \mathrm{mg} /$ $\mathrm{kg}$ (Figure $1 \mathrm{~B}$ ). AM281 is a $\mathrm{CB}_{1} \mathrm{R}$ antagonist/inverse agonist; however, the $C_{1}$ R neutral antagonist NESSO327 (21) also significantly shortened recovery time after isoflurane anesthesia (Figure 1C). Similar to the case with isoflurane, AM281 (3 mg/kg, i.p.) significantly shortened recovery time in rats anesthetized with sevoflurane (Figure 1D). Based on these results, isoflurane anesthesia and an i.p. injection of AM281 (3 mg/ $\mathrm{kg})$ were used in all subsequent experiments unless otherwise stated.

Next, we investigated the hypothesis that systemic AM281 accelerates arousal by a direct blockade of $\mathrm{CB}_{1} \mathrm{R}$ in the wake-promoting Pef, the sleep-promoting VLPO, or both. This hypothesis is unlikely to be supported, as no significant effects on recovery time were detected after bilateral microinjection of AM281 (0.1 $\mu \mathrm{g} / 0.3$ $\mu \mathrm{l} /$ side) into either the Pef (Figure 1E and Supplemental Figure 4) or the VLPO (Figure 1F and Supplemental Figure 4). Because the $\mathrm{DMH}$ plays an essential role in the integration of sleep and wakefulness (9-12), we then probed whether intra-DMH application of AM281 could mimic the effects of systemically injected AM281. Indeed, bilateral intra-DMH application of AM281 (0.1 $\mu \mathrm{g} / 0.3 \mu \mathrm{l} /$ side) (Supplemental Figure 4) significantly shortened recovery time (Figure 1G).

Anesthetics enhance eCB signaling at $D M H$ glutamatergic but not GABAergic synapses. To reveal how anesthetics modulate $\mathrm{eCB}$ signaling in the DMH, we performed whole-cell patch clamp on hypothalamic slices. We identified two types of DMH neurons with distinct electrical fingerprints, i.e., neurons with or without 
A
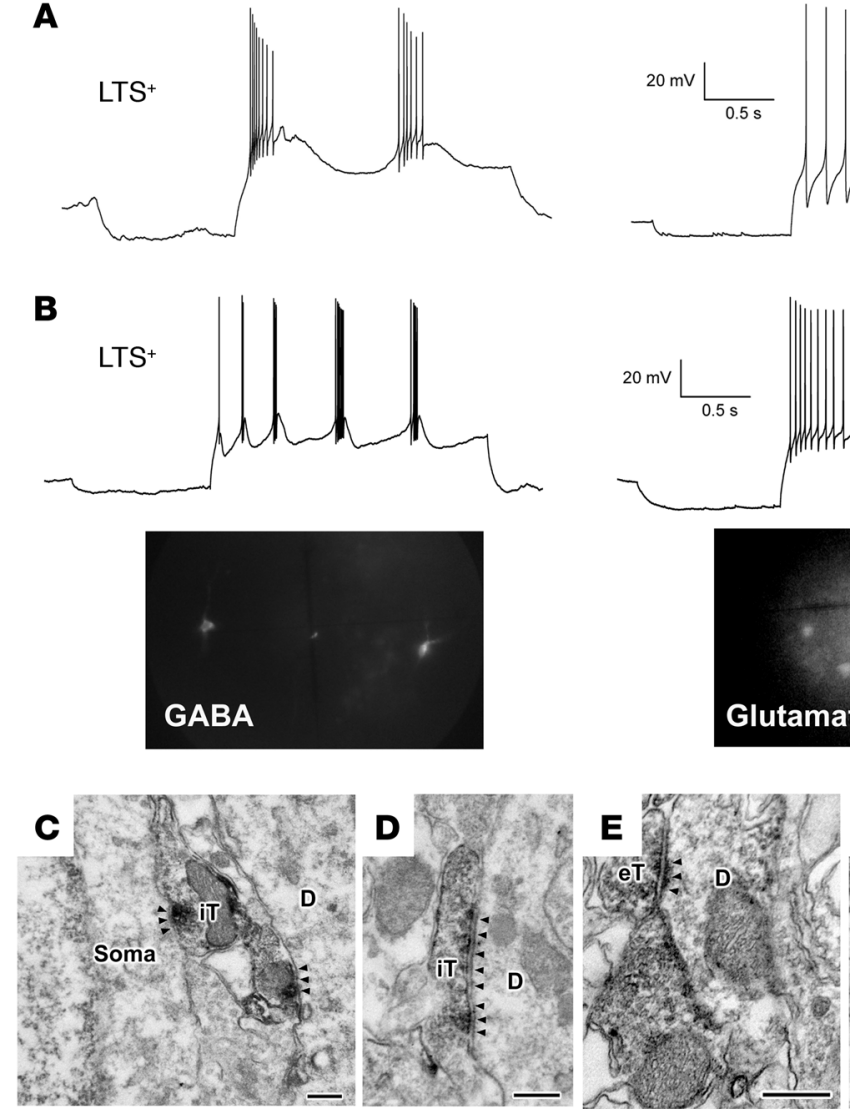

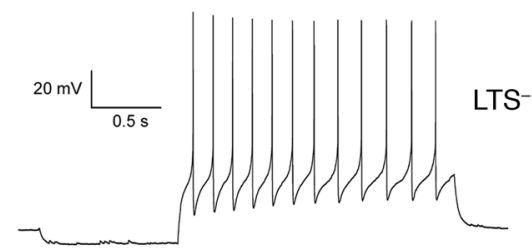

LTS $^{-}$

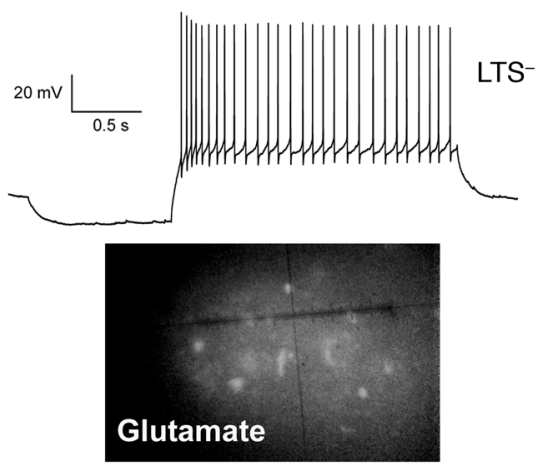

LTS- $^{-}$

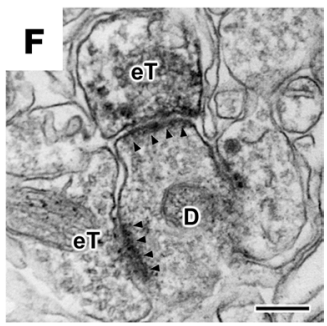

Figure 2. Electrophysiological and ultrastructural characteristics of DMH GABAergic and glutamatergic neurons. (A) Sample current clamp traces ( $>20$ repetitions) show two distinct types of DMH neurons with or without low-threshold spikes, i.e., LTS+ or LTS-, respectively, elicited when the membrane is depolarized from negative potentials. (B) Sample current clamp traces $(>20$ repetitions) of LTS+ or LTS- (top) were recorded from fluorescent $D M H$ neurons (bottom photos) of GABAergic (CABA) and glutamatergic neurons (Glutamate) from GAD67-GFP and vGLUT2iCreERT2 mutant mice, respectively. (C-F) Electron microscopic images (3 mice) show $\mathrm{CB}_{1} \mathrm{R}$ immunoreactive peroxidase reaction product in DMH GABAergic axonal terminals ( $\mathrm{i}$ i in $\mathbf{C}$ and $\mathbf{D}$ ) or glutamatergic axonal terminals (eT in $\mathbf{E}$ and $\mathbf{F}$ ). Black arrowheads identify symmetric and asymmetric synaptic contacts of presumed GABAergic and glutamatergic nature, respectively, with the neuronal soma profile (Soma) or dendritic profile (D). Scale bars: $0.25 \mu \mathrm{m}$. low-threshold spikes (LTS), referred as $\mathrm{LTS}^{+}$or $\mathrm{LTS}^{-}$neurons, respectively (22) (Figure 2A). Glutamic acid decarboxylase 67 (GAD67) is a GABA biosynthesis enzyme, so we employed transgenic mice expressing EGFP selectively in GAD67-synthesizing neurons (i.e., GAD67-GFP mice) (23) to identify GABAergic neurons under fluorescence microscopy. To identify DMH glutamatergic neurons under fluorescence microscopy, we injected a double-floxed (DIO) Cre recombinase-dependent adenoassociated virus (AAV) vector fused with mCherry to form AAV-mCherry into the DMH of mice with knockin of improved CreERT2 (iCreERT2) into the Vglut2 locus (hereafter referred to as vGLUT2-iCreERT2 mice; Supplemental Figures 5 and 6). Fluorescent DMH cells in GAD67-GFP and vGLUT2-iCreERT2 mutant mice were $\mathrm{LTS}^{+}$and $\mathrm{LTS}^{-}$neurons, respectively (Figure 2B), thus representing DMH GABAergic and glutamatergic neurons, respectively.

The $\mathrm{DMH}$ contains a moderate density of $\mathrm{CB}_{1} \mathrm{R}$-immunoreactive axons/terminals (24). Notably, our electron microscopic study showed that approximately $32 \%$ of DMH inhibitory synapses and $8 \%$ of $\mathrm{DMH}$ excitatory synapses were $\mathrm{CB}_{1} \mathrm{R}$-immunopositive (Figure 2, C-F). We then recorded depolarization-induced suppression of inhibition (DSI) or excitation (DSE) at DMH GABAergic or glutamatergic synapses, respectively, because both DSI and DSE are specifically associated with eCB signaling (17, 25, 26). Bath application of isoflurane did not significantly affect DSI in either LTS $^{+}$or LTS ${ }^{-}$neurons in the DMH (Figure 3, A, B, and $\mathrm{E})$, suggesting that isoflurane does not significantly affect eCB signaling at GABAergic synapses onto either GABAergic or glu- tamatergic DMH neurons. In contrast, isoflurane significantly increased DSE in both $\mathrm{LTS}^{+}$and $\mathrm{LTS}^{-}$neurons (Figure 3, C-E), suggesting that isoflurane significantly increased eCB signaling at glutamatergic synapses in both GABAergic and glutamatergic $\mathrm{DMH}$ neurons. eCB activation of glutamatergic presynaptic $\mathrm{CB}_{1} \mathrm{R}$ inhibits presynaptic release of glutamate, inducing deactivation (i.e., inhibition) of postsynaptic neurons $(17,18)$. We therefore hypothesized that general anesthesia would suppress both LTS $^{+}$and LTS $^{-}$neurons in the DMH in a CB R-dependent manner. Indeed, bath application of either isoflurane or sevoflurane onto hypothalamic slices significantly reduced the firing rate of both $\mathrm{LTS}^{+}$and LTS $^{-}$neurons, which returned to pretreatment levels after washout of isoflurane (Figure $3 \mathrm{~F}$ ) or sevoflurane (Supplemental Figure 7A). In the presence of AM281, however, isoflurane did not significantly affect the firing rate of either LTS $^{+}$or LTS $^{-}$neurons (Supplemental Figure 7B).

Blocking $C B_{1} R$ at DMH glutamatergic synapses accelerates arousal through VLPO and Pef. Based on our electrophysiological findings, we reasoned that if systemic AM281 shortened recovery time via increased release of presynaptic glutamate but not GABA in the DMH, then intra-DMH application of the glutamate $N$-methyl-D-aspartate receptor (NMDAR) antagonist MK801, but not the GABA receptor (GABAR) antagonist bicuculline, would block the effects of systemic AM281. As expected, bilateral intra-DMH administration of MK801 (0.005 $\mu \mathrm{g} / 0.3 \mu \mathrm{l} / \mathrm{side})$, but not bicuculline $(0.2 \mu \mathrm{g} / 0.3 \mu \mathrm{l} /$ side) (Supplemental Figure 4), abolished the shortened recovery time induced by systemic AM281 (Figure 4, A and B). 
A

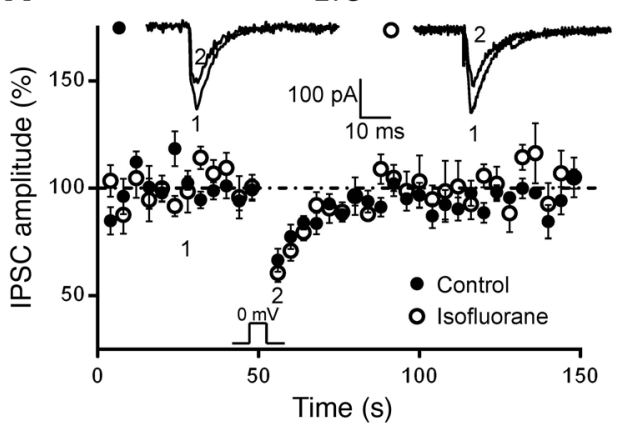

C

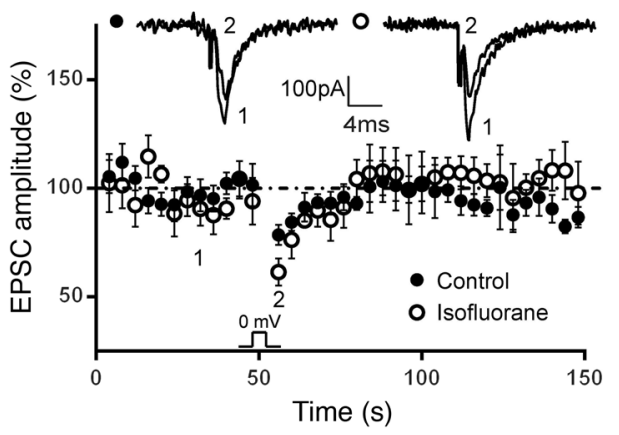

B

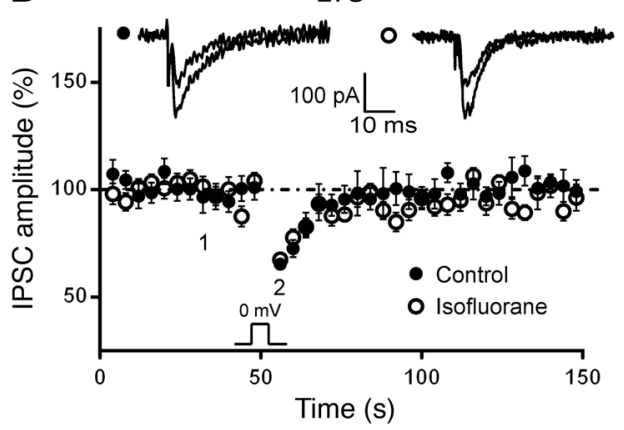

D

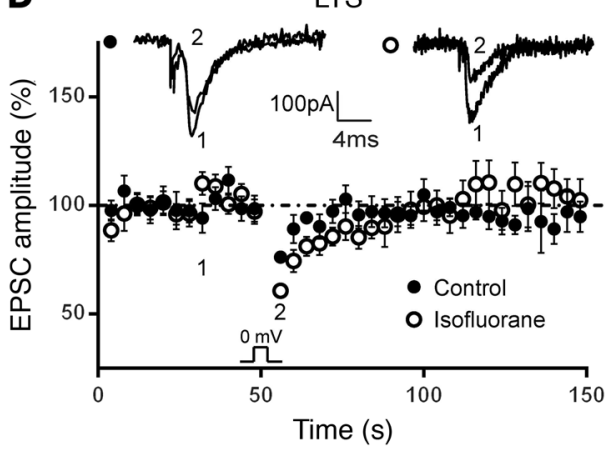

Figure 3. Isoflurane-mediated increase of eCB signaling in DMH glutamatergic but not GABAergic synapses. (A-E) Plots of normalized IPSC (A and B) or EPSC amplitude (C and $\mathbf{D}$ ) and summary histograms (E) show that the DSI magnitude of DMH LTS+ and LTS- neurons does not significantly change following bath application of isoflurane (Iso) as compared with baseline (Con) (E: A, $P=$ 0.1333; B, $P=0.8729$ ), which, however, significantly increases DSE magnitude of both $\mathrm{LTS}^{+}$and LTS- neurons. Representative IPSC and EPSC traces are shown above each plot. The summarized data in $\mathbf{E}$ represent the first time point after depolarization of the cell, which is marked with " 2 " in A-D. (F) The representative traces (left) and summary histograms (right) show that relative to baseline (Con), bath application of isoflurane induces rapid hyperpolarization of the membrane potential and significantly decreases of the firing rate of both LTS+ and LTS neurons in the DMH, which return to the baseline levels after washout (Wash). All summary graphs show mean \pm SEM; $n$, number of rats recorded in each group. ${ }^{*} P<0.05$ and ${ }^{*} P<0.01$ vs. Con, Student's $t$ test (E) or Tukey's post-hoc test after 1-way ANOVA (F: LTS $+F_{2,12}$ $=5.669, P<0.05 ;$ LTS $^{-}, F_{2,3212}=$ $8.517, P<0.01)$
$\mathbf{F}$

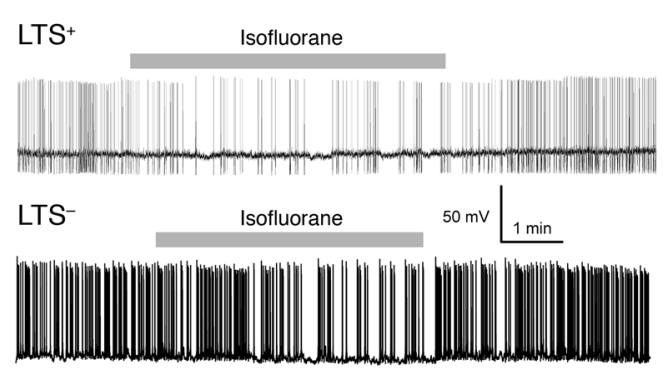

$\mathrm{LTS}^{+}$

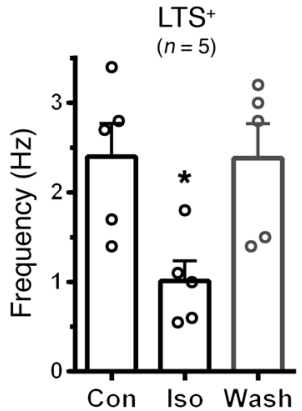

LTS-

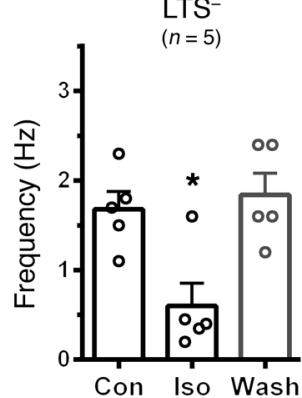

The DMH sends GABAergic and glutamatergic axons innervating the VLPO and Pef, respectively (9-13). If systemic AM281 selectively increases release of presynaptic glutamate but not GABA in the DMH, both GABAergic DMH-VLPO and glutamatergic DMH-Pef projections should be activated. We therefore hypothesized that blocking either VLPO GABAergic or Pef glutamatergic synaptic neurotransmission would abolish the shortened recovery time induced by systemic AM281. In support of this hypothesis, bilateral intra-VLPO injection of bicuculline (0.2 $\mu \mathrm{g} / 0.3 \mu \mathrm{l} /$ side) (Supplemental Figure 4) or bilateral intra-Pef injection of MK801 (0.005 $\mu \mathrm{g} / 0.3 \mu \mathrm{l} / \mathrm{side}$ ) (Supplemental Figure 4), doses that did not significantly affect recovery time on their own, prevented systemic AM281 from shortening recovery time (Figure 4, C and D). Interestingly, systemic injection of either bicuculline ( $0.5 \mathrm{mg} / \mathrm{kg}$, i.p.) or MK801 (0.02 mg/kg, i.p.), at doses that did not significantly affect recovery time on their own, also prevented sys- 
A

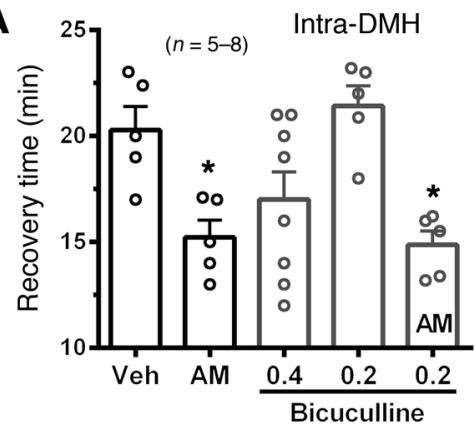

C

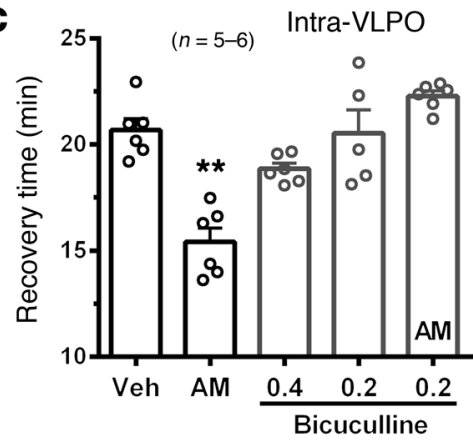

E

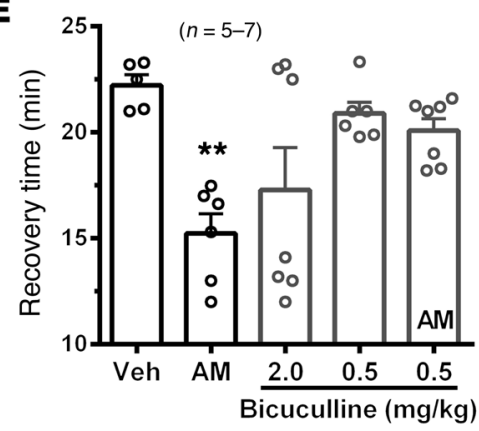

B

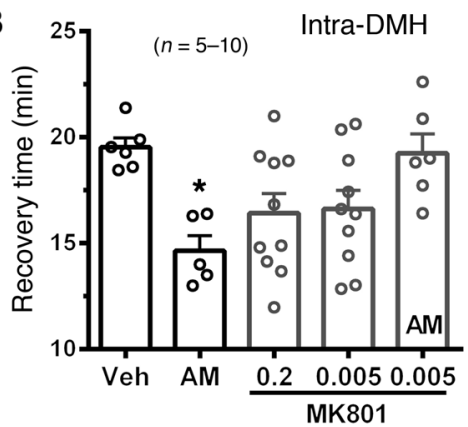

D

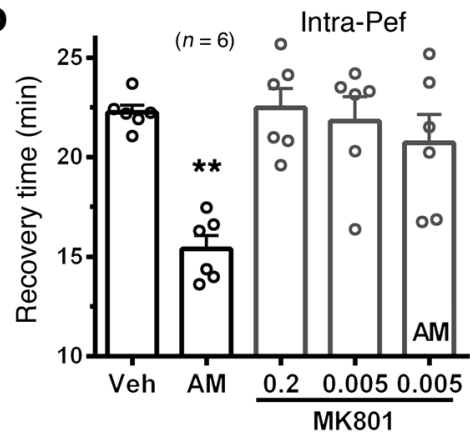

$\mathbf{F}$

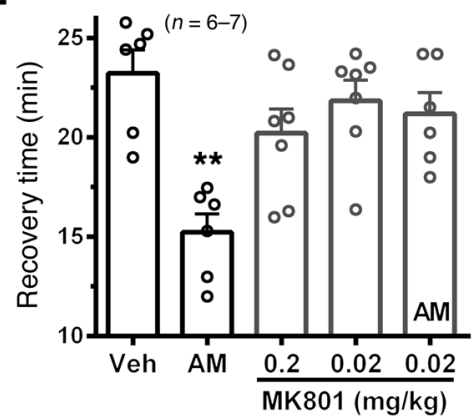

Figure 4. Blockade of $\mathrm{CB}_{1} \mathrm{R}$ at $\mathrm{DMH}$ glutamatergic synapses accelerates arousal through VLPO and Pef. Both high and low doses of the GABAR antagonist bicuculline and the NMDAR antagonist MK801 were used in order to reveal their dose-response effects on arousal after anesthesia. (A-D) Relative to vehicle (Veh), AM281 (AM, $3.0 \mathrm{mg} / \mathrm{kg}$, i.p.) significantly shortens recovery time, which is not significantly affected by bilateral intra-DMH microinjection of bicuculline (A: $0.2 \mu \mathrm{g} /$ side/ injection). Bilateral intra-DMH microinjection of MK801 (B: $0.005 \mu \mathrm{g} / \mathrm{side} /$ injection), intra-VLPO microinjection of bicuculline (C: $0.2 \mu \mathrm{g} / \mathrm{side} /$ injection), and intra-Pef microinjection of MK801 (D: $0.005 \mu \mathrm{g} /$ side/injection) which do not significantly affect recovery time on their own - prevents AM281 (3 mg/kg, i.p.) from shortening recovery time. (E and $\mathbf{F}$ ) Relative to Veh, AM significantly shortens recovery time, which is blocked by an i.p injection of $0.5 \mathrm{mg} / \mathrm{kg}$ bicuculline (E) or $0.02 \mathrm{mg} / \mathrm{kg}$ MK801 (F) that does not significantly affect recovery time on its own. All summary graphs show mean $\pm S E M ; n$, number of rats in each group. ${ }^{*} P<0.05$ and ${ }^{* *} P<0.01$ vs. Veh, Tukey's post-hoc test after 1-way ANOVA (A: $F_{423}=22.6$, $P<0.01 ; \mathbf{B}: F_{4,32}=29.101, P<0.01 ;$ C: $F_{4.24}=32.101, P<$ $0.01 ; \mathbf{D}: F_{4,25}=15.025, P<0.01 ; \mathbf{E}_{1} F_{4,26}=34.053, P<0.01$ F: $F_{4,27}=19.273, P<0.01$. temic AM281 from shortening recovery time (Figure 4, E and F), indicating important contributions of either enhanced GABAergic DMH-VLPO projection or enhanced glutamatergic DMH-Pef projection to eCB modulation of arousal from anesthesia.

$C B_{1} R$ deletion from PFC glutamatergic neurons accelerates arousal. Our electrophysiological and behavioral results together suggest that activation of $\mathrm{CB}_{1} \mathrm{R}$ at $\mathrm{DMH}$ glutamatergic input participates in arousal from anesthesia. Because DMH neurons receive strong innervation directly from glutamatergic neurons in the prelimbic and infralimbic areas of the PFC (14), we examined the contributions of $\mathrm{CB}_{1} \mathrm{R}$ at glutamatergic $\mathrm{PFC}-\mathrm{DMH}$ projection to arousal by taking advantage of conditional mutagenesis to selectively delete $C B 1 R$ from glutamatergic neurons. Glutamatergic neurons in the cerebral cortex, including the PFC, contain the glutamate transporter vGLUT1 (27). CB1R-floxed mice $\left(C B 1 R^{f l f l}\right)$ (28) were crossed with vGLUT1-iCreERT2 mutant mice with knockin of iCreERT2 into the Vglut1 locus (Supplemental Figures 8 and 9) to generate a vGLUT1-CB R-KO mouse line. Electron microscopy studies confirmed the absence of $\mathrm{CB}_{1} \mathrm{R}$ immunoreactivity selectively in vGLUT1-immunopositive glutamatergic terminals in the $\mathrm{DMH}$ (Figure 5, A-C), suggesting an inducible deletion of $C B 1 R$ from glutamatergic axon terminals of the PFC-DMH projection in vGLUT1- $\mathrm{CB}_{1} \mathrm{R}-\mathrm{KO}$ mice. Relative to vGLUT1-CB $\mathrm{R}-\mathrm{WT}$ mice, vGLUT1-CB $\mathrm{R}-\mathrm{KO}$ littermates recovered significantly faster after anesthesia (Figure 5D).

These results are not conclusive, however, because of vGLUT1's widespread expression in glutamatergic neurons throughout the cerebral cortex, including the PFC. To overcome this potential limitation, we further examined the effects of selective deletion of $C B 1 R$ from glutamatergic PFC neurons on arousal from anesthesia. AAV-CaMKII-iCre-GFP was injected bilaterally into prelimbic and infralimbic PFC in CB1R-floxed mice and their WT littermates (Supplemental Figure 10), i.e., PFC- $\mathrm{CB}_{1} \mathrm{R}$-flox and PFC- $\mathrm{CB}_{1} \mathrm{R}-\mathrm{WT}$ littermates. Electron microscopy study confirmed the absence of $C_{1} R$ immunoreactivity in many vGLUT1-immunopositive glutamatergic terminals in the DMH (Figure 5, E-G), suggesting an effective deletion of $C B 1 R$ in axon terminals of the glutamatergic $\mathrm{PFC}-\mathrm{DMH}$ projection. Relative to PFC-CB $\mathrm{R}-\mathrm{WT}$ mice, $\mathrm{PFC}-\mathrm{CB}_{1} \mathrm{R}$-flox littermates recovered significantly faster after anesthesia (Figure $5 \mathrm{H}$ ).

Our initial findings that intra-Pef application of the $\mathrm{CB}_{1} \mathrm{R}$ antagonist AM281 did not significantly affect arousal from anesthesia suggest that $\mathrm{CB}_{1} \mathrm{R}$ in the glutamatergic $\mathrm{DMH}$-Pef projection 
A

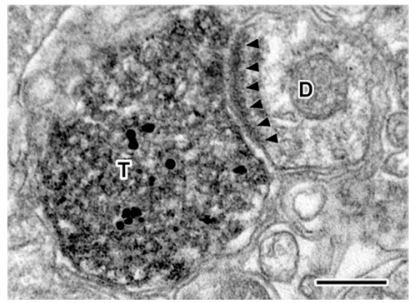

vGLUT1-CB,R-WT

E

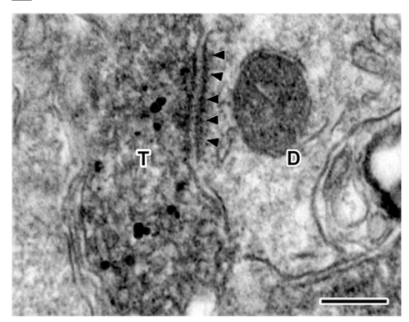

PFC-CB, R-WT

I

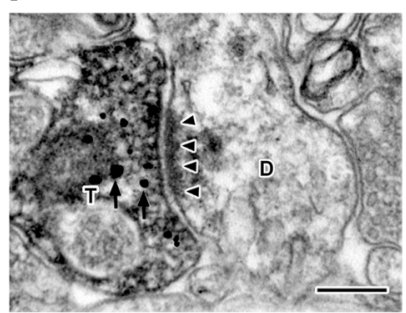

vGLUT2-CB ${ }_{1}$ R-WT

M

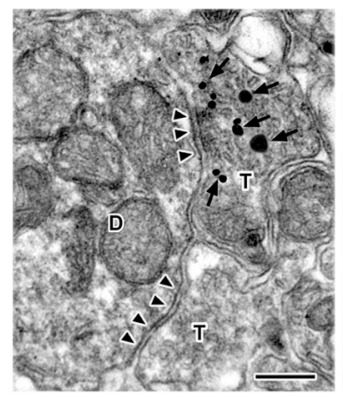

GAD65-CB, R-WT
B

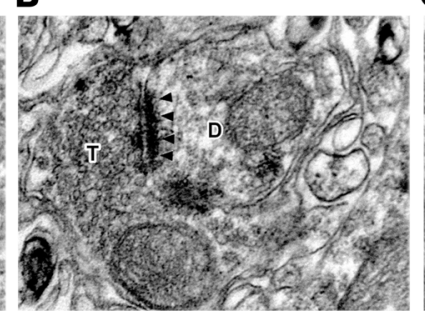

vGLUT1-CB,R-KO

$\mathbf{F}$

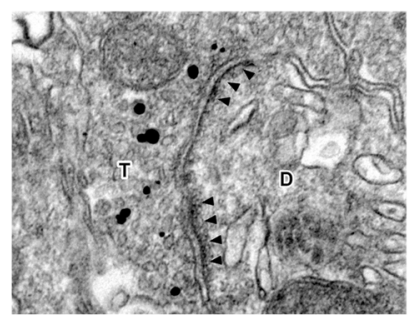

G

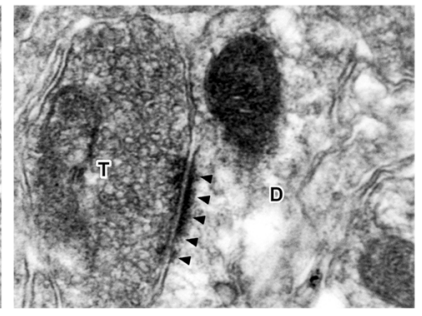

PFC-CB, R-KO

J

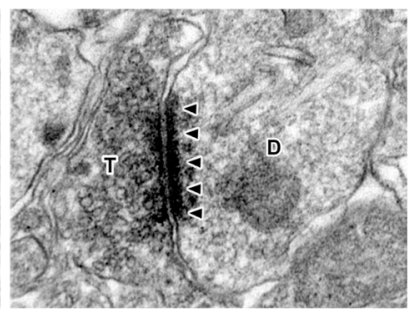

vGLUT2-CB, R-KO

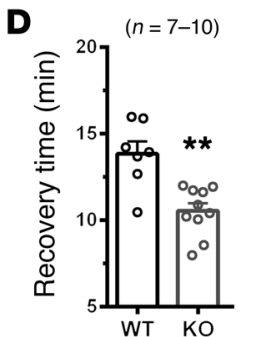

vGLUT1-CB, $R$

H $\quad(n=10-11)$

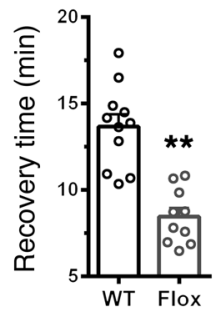

PFC-CB, $R$

$\mathbf{L}$

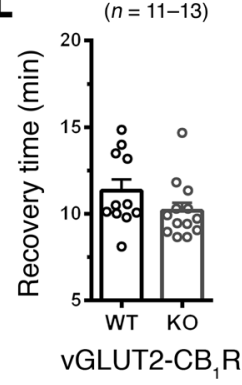

$\mathbf{N}$

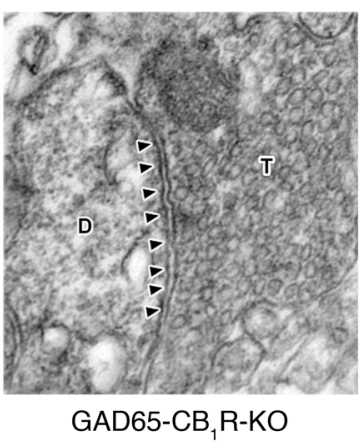

o

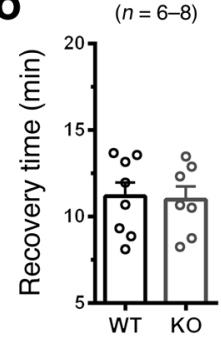

GAD65-CB ${ }_{1}$

Figure 5. CB1R deletion from PFC glutamatergic neurons accelerates arousal. (A-C, E-G, I-K, M, and $\mathbf{N}$ ) In these electron microscopic images (3-4 mice in each genotype), the presumed glutamatergic and GABAergic nature of an axon terminal (T) is established by the presence of asymmetric (A-C, $\mathbf{E}-\mathbf{C}$, and $\mathbf{I}-\mathbf{K}$ ) or symmetric ( $\mathbf{M}$ and $\mathbf{N}$ ) synaptic contact (black arrowheads) with a dendritic (D) profile. $\mathrm{CB}_{1} \mathrm{R}$-immunoreactive (CB, $\mathrm{R}$-ir) silver grains are in both vGLUT1-ir glutamatergic terminals in the DMH of vGLUT1-CB,R-WT (A) or PFC-CB,R-WT mice (E) or the Pef of vGLUT2-CB,R-WT mice (I); and in VGLUT1immunonegative (VGLUT1-in) (C and G) or vGLUT2-in (K) glutamatergic terminals in the DMH of vGLUT1-CB,R-KO (C) or PFC-CB, R-KO mice (G) or the Pef of vGLUT2-CB, R-KO mice (K); but not in vGLUT1-ir (B and F) or vGLUT2-ir (J) glutamatergic terminals in the DMH of vGLUT1-CB, R-KO (B) or PFC-CB,R-KO mice $(\mathbf{F})$ or the Pef of vGLUT2-CB, R-KO mice (J). Similarly, $\mathrm{CB}_{1} \mathrm{R}$-ir silver grains are in GABAergic terminals in the DMH of CAD65-CB $R$-WT mice (M) but not in CAD65-CB,R-KO mice (N). Scale bars: $0.25 \mu \mathrm{m}$. (D, H, L, and $\mathbf{O})$ A significant decrease in recovery time is detected in vGLUT1-CB,R-KO (D) and PFC-CB,R-KO mice $(\mathbf{H})$, but not vGLUT2-CB, R-KO $(\mathrm{L}: P=0.151)$ and $\mathrm{CAD65}-\mathrm{CB}_{1} \mathrm{R}-\mathrm{KO}$ mice $(\mathbf{N}: P=0.8659)$, relative to corresponding WT littermates. All summary graphs show mean $\pm \mathrm{SEM} ; n$, number of mice in each group. ${ }^{* *} P<0.01 \mathrm{vs}$. WT, Student's $t$ test.

is not required for arousal after anesthesia. To confirm these findings, we further studied the effects of selective deletion of $C B 1 R$ from vGLUT2-containing glutamatergic neurons in the hypothalamus (27). The CB1R-floxed mice (28) were crossed with vGLUT2iCreERT2 mice as described above to generate the vGLUT2-
$\mathrm{CB}_{1} \mathrm{R}-\mathrm{KO}$ mouse line. Electron microscopy study confirmed the absence of $\mathrm{CB}_{1} \mathrm{R}$ immunoreactivity in vGLUT2-immunopositive glutamatergic terminals in the Pef (Figure 5, I-K). vGLUT2- $\mathrm{CB}_{1} \mathrm{R}-$ KO and vGLUT2-CB $\mathrm{CB}_{1}$-WT littermates showed similar recovery time after anesthesia (Figure 5L). 
A

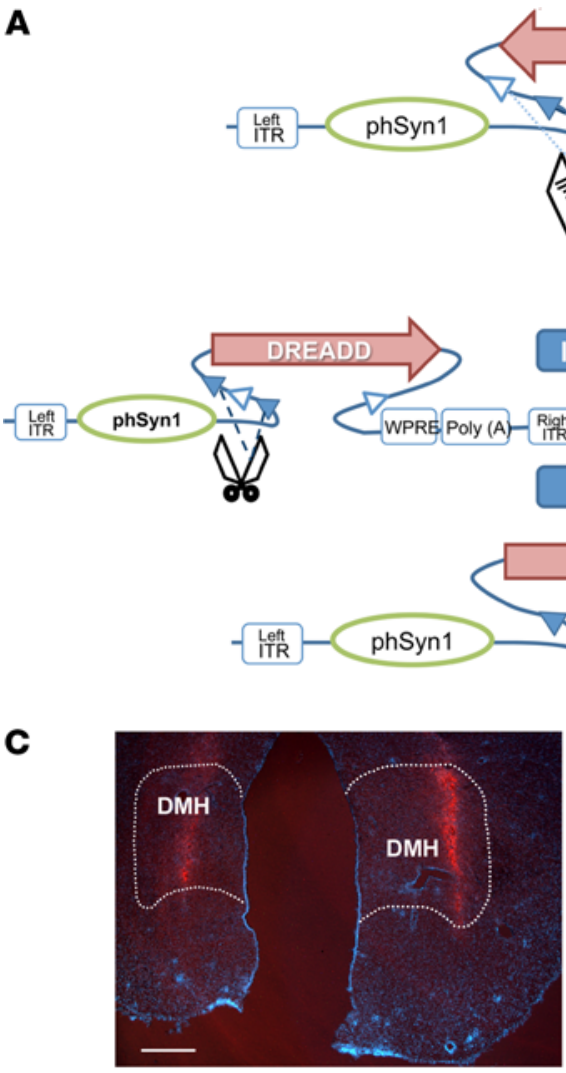

E

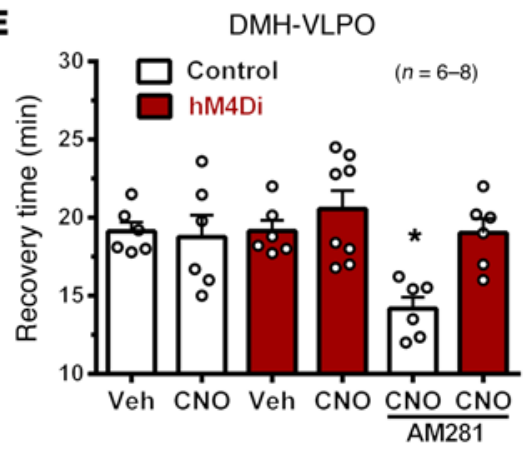

बवसבप्रव

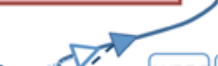

-

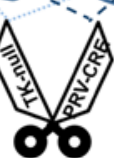

Inversion

पर

(n)
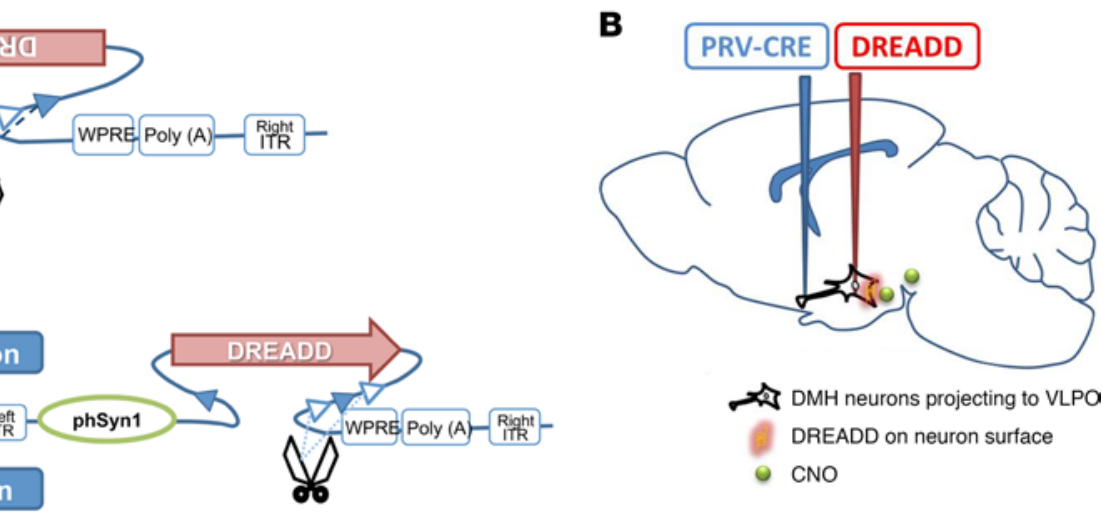

B
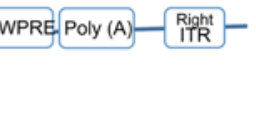

.

(1) 
A

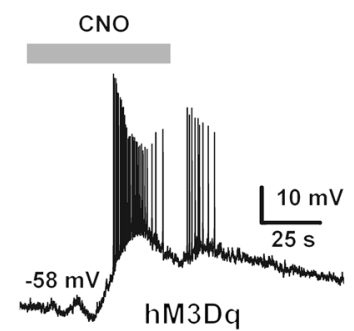

D

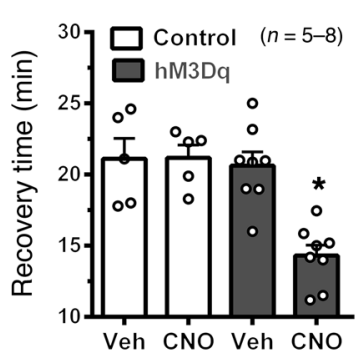

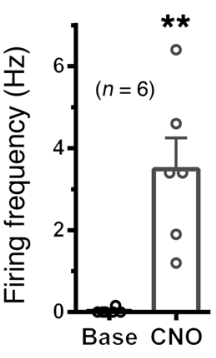

E

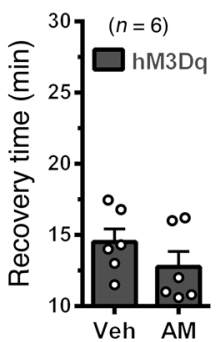

B

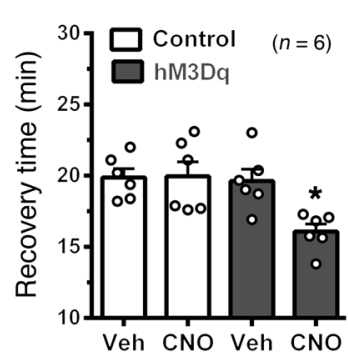

$\mathbf{F}$

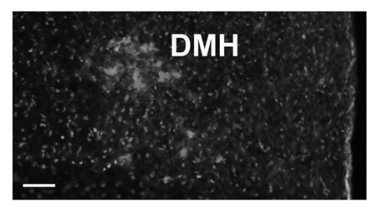

I
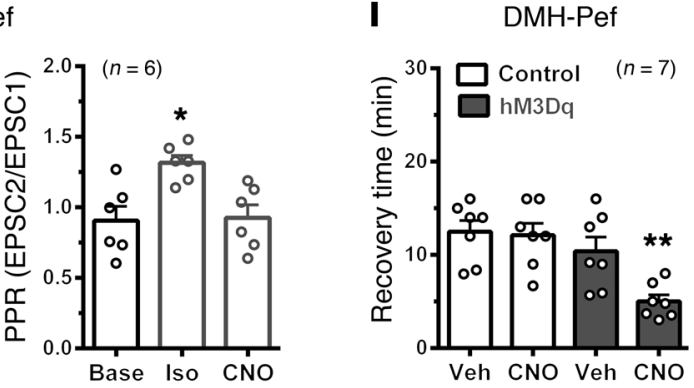

H

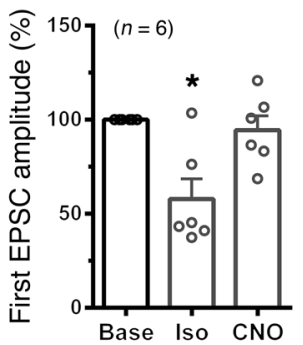

DMH-Pef

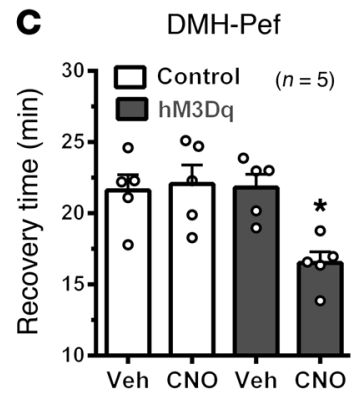

G

DMH-Pef
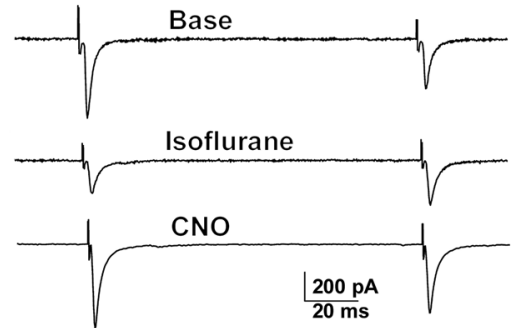

J PFC-DMH

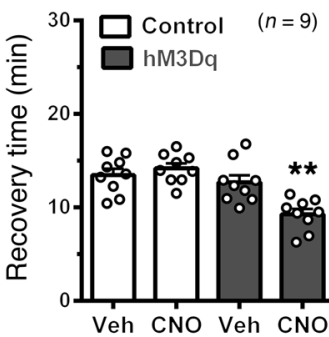

Figure 7. PFC-DMH-Pef/VLPO activation enhances arousal. (A) A sample trace and summary histogram show that CNO induces rapid depolarization of membrane potential and increases firing rate of DMH neurons with red mCherry fluorescence after intra-VLPO and intra-DMH injection of PRV-Cre and AAV-hM3Dq-mCherry (hM3Dq), respectively. (B-E, I, and J) Rats (B-E), vGLUT2-iCreERT2 mice (I), or vGLUT1-iCreERT2 mice (J) received an i.p. (B-E), intra-Pef (I), or intra-DMH injection (J) of vehicle or CNO 1-2 weeks after receiving intra-DMH (B and C) or intra-PFC (D, E, I, and J) injection of AAV-mCherry (Control) or AAV-hM3Dq-mCherry (hM3Dq) with or without intra-VLPO (B), intra-Pef (C), or intra-DMH (D and E) injection of PRV-Cre. The hM3Dq animals receiving CNO show a significant decrease in recovery time $(P<0.05$ or $P<0.01)$ relative to each of the other 3 groups $(\mathbf{B}-\mathbf{D}$, I, and J), but the hM3Dq animals injected with AM281 (AM; $3 \mathrm{mg} / \mathrm{kg}$, i.p.) or its vehicle do not show a significant difference $(P=0.3601)$ in recovery time $(\mathrm{E})$. (F-H) After intra-DMH injection of hM3Dq into vGLUT2-iCreERT2 mice, the DMH neurons containing both vGLUT2-driven iCre and mCherry are visible as fluorescent-labeling cells. (F: scale bar: $100 \mu \mathrm{m}$ ). Sample traces $(\mathbf{C})$ and summary histograms $(\mathbf{H})$ show that with patch clamp recording of Pef neurons and stimulation of DMH input axons, isoflurane reduces and increases the first EPSC amplitude and PPR, respectively, which are reversed by CNO. All summary graphs show mean \pm SEM; $n$, number of animals in each group. ${ }^{*} P<0.05$ and ${ }^{* *} P<0.01$ vs. baseline or Veh, Student's $t$ test (A and $\left.\mathbf{E}\right)$ or Tukey's post-hoc test after 1-way ANOVA (B: $F_{3.20}=5.8893, P<0.01 ; \mathbf{C}: F_{3,16}=6.328, P<0.01 ; \mathbf{D}: F_{3,22}=12.65, P<0.01 ; \mathbf{H}:$ left, $F_{2,15}=9.045, P<0.01 ;$ right, $\left.F_{2,15}=7.492, P<0.01 ; \mathrm{I}: F_{3,16}=8.044, P<0.01 ; \mathrm{J}: F_{3,32}=11.77, P<0.01\right)$.

immunoreactivity in GABAergic axon terminals in the DMH of GAD65-CB $\mathrm{R}-\mathrm{KO}$ mice (Figure 5, $\mathrm{M}$ and N). GAD65-CB ${ }_{1} \mathrm{R}-\mathrm{KO}$ and GAD65- $\mathrm{CB}_{1} \mathrm{R}-\mathrm{WT}$ littermates showed similar recovery times after anesthesia (Figure 5O), thus supporting our electrophysiological and behavioral data obtained in WT rats.

PFC-DMH-Pef/VLPO inactivation counteracts AM281's effects on arousal. Our behavioral and electrophysiological studies suggest that AM281-mediated activation of PFC-DMH, DMH-Pef, and DMH-VLPO projections accelerates arousal from anesthesia. Thus, selective inactivation of these projections could counteract AM281's effects. To examine this hypothesis, we employed a combination of designer receptors exclusively activated by designer drugs (DREADD) technology and the pseudorabies virus (PRV) vector expressing Cre recombinase (PRV-Cre). To generate a DREADD, we mutated muscarinic receptors to be activated by clozapine $\mathrm{N}$-oxide (CNO) but not by their natural ligands (29-31). DREADD was then delivered by an AAV expressing a doubly floxed inverted open reading frame, which was fused with mCherry to form AAV-DREADD-mCherry. Thus, DREADD expression could be monitored, and stable transgene inversion was achieved with the FLEX Switch (Figure 6A) (32). After intra-VLPO injection, PRV-Cre entered axonal terminals and then underwent retrograde transport $(33,34)$ to DMH neurons (Figure 6B). As a thymidine kinase gene-null viral vector, the PRV-Cre used here 
is unable to replicate in neurons or to be transmitted trans-synaptically and does not produce detectable deleterious effects on infected neurons or animals (33). When AAV-DREADD-mCherry was injected into the $\mathrm{DMH}, \mathrm{mCherry}$ was detected in those $\mathrm{DMH}$ neurons projecting to the VLPO that had received PRV-Cre injection (Figure 6, B and C).

To inactivate DMH-VLPO projection, we employed $\mathrm{G}_{\mathrm{i} / \mathrm{o}}$-coupled hM4Di receptors, whose activation by CNO stimulates $G$ protein-coupled inwardly rectifying potassium channels, resulting in membrane hyperpolarization and subsequent neuronal silencing (29-31). Rats received intra-VLPO injection of PRV-Cre and intra-DMH injection of AAV-hM4Di-mCherry (Supplemental Figure 12). Patch clamp recording revealed that bath application of CNO to hypothalamic slices significantly decreased the firing rate of mCherry-labeled DMH neurons (Figure 6D), suggesting that CNO activation of AAV-hM4Di-mCherry inactivates the DMH neurons innervating the VLPO. As expected, an i.p. injection of $\mathrm{CNO}$ in these rats prevented systemic AM281 from shortening recovery time (Figure 6E). Similar results were observed in rats receiving intra-Pef injection of PRV-Cre and intra-DMH injection of AAV-hM4Di-mCherry (Figure 6F and Supplemental Figure 13) or intra-DMH injection of PRV-Cre and intra-PFC (i.e., prelimbic and infralimbic areas of the PFC) injection of AAV-hM4DimCherry (Figure 6G and Supplemental Figure 14).

PFC-DMH-Pef/VLPO activation enhances arousal. We next examined whether selective activation of DMH-VLPO, DMHPef, or PFC-DMH projection could mimic the effects of AM281 on arousal from anesthesia. To activate DMH-VLPO projection, we employed Gq-coupled hM3Dq receptors, as their activation by CNO induces membrane depolarization and subsequent neuronal activation (29-31). Rats received intra-VLPO injection of PRV-Cre and intra-DMH injection of AAV-hM3Dq-mCherry (Supplemental Figure 12). Patch clamp recording revealed that bath application of $\mathrm{CNO}$ to hypothalamic slices significantly increased the firing rate of mCherry-labeled DMH neurons (Figure 7A), suggesting CNO activates DMH-VLPO projection. As expected, an i.p. injection of CNO significantly shortened recovery time (Figure 7B). Similar results were found in rats receiving either intra-Pef injection of PRV-Cre and intra-DMH injection of AAV-hM3Dq-mCherry (Figure $7 \mathrm{C}$ and Supplemental Figure 13) or intra-DMH injection of PRVCre and intra-PFC injection of AAV-hM3Dq-mCherry (Figure 7D and Supplemental Figure 14). However, rats treated with AM281 (3 $\mathrm{mg} / \mathrm{kg}$, i.p.) or its vehicle after receiving intra-DMH injection of PRV-Cre and intra-PFC injection of AAV-hM3Dq-mCherry did not show a significant difference in recovery time (Figure $7 \mathrm{E}$ and Supplemental Figure 14), indicating the bottom or floor effects produced by AM281 injection or DREADD-mediated activation of PFC-DMH projection.

These results are inconclusive for two reasons. First, because the same DMH neurons innervate both the Pef and other brain regions (13), CNO stimulation of these neurons could activate the Pef and other brain regions. Second, while approximately $59 \%$ of DMH-Pef projections were glutamate immunoreactive (13), CNO activation of DMH neurons retrogradely labeled by intra-Pef-injected PRV-Cre could activate both glutamatergic and non-glutamatergic DMH neurons. To overcome these potential problems, we studied the effects of selective activation of axon terminals of the glutamatergic DMH-Pef projections. When $\mathrm{AAV}-\mathrm{hM} 4 \mathrm{Di}$ was injected into a brain region, it entered neuronal cell bodies and then was anterogradely transported to their axon terminals where CNO was administered to activate hM4Di, hence inhibiting presynaptic release of neurotransmitters $(35,36)$. After intra-DMH injection of AAV-hM3Dq-mCherry in vGLUT2iCreERT2 mice, mCherry was detected in DMH cells (Figure 7F), suggesting iCre-mediated inversion and expression of the hM3Dq in glutamatergic neurons. Ten days later, the mice were killed for patch clamp recordings of glutamatergic DMH-Pef projection. Bath application of isoflurane onto hypothalamic slices significantly reduced the amplitude of the first excitatory postsynaptic currents (EPSCs) and significantly increased the paired pulse ratio (PPR), changes that were reversed by $\mathrm{CNO}$ (Figure 7, G and $\mathrm{H}$ ). In another group of vGLUT2-iCreERT2 mice receiving intra-DMH injection of AAV-hM3Dq-mCherry, bilateral intra-Pef injection of CNO significantly shortened recovery time (Figure 7I and Supplemental Figure 15). Collectively, the morphological, electrophysiological, and behavioral data shown in Figure 7, F-I, suggest that after intra-DMH injection in vGLUT2-iCreERT2 mice, hM3Dq was expressed in DMH glutamatergic neurons and then anterogradely transported to their axon terminals in the Pef, where CNO activation of presynaptic hM3Dq increased release of glutamate at glutamatergic DMH-Pef synapses. As expected, we also observed that bilateral intra-DMH injection of CNO significantly shortened recovery time in vGLUT1-iCreERT2 mice receiving bilateral intra-PFC injection of AAV-hM3Dq-mCherry 2 weeks before CNO injection (Figure 7J and Supplemental Figure 16).

\section{Discussion}

It is unknown whether eCB signaling participates in arousal from general anesthesia. We observed here that increased eCB signaling by the eCB degradation inhibitor JZL195 delayed arousal after general anesthesia, whereas the $\mathrm{CB}_{1} \mathrm{R}$ antagonists AM281 and NESSO327 accelerated arousal. Application of AM281 into the DMH mimicked the effects of systemic injection, suggesting that decreased eCB signaling in the $\mathrm{DMH}$ participates in arousal from anesthesia. GABAergic SCN-DMH projection is required for circadian rhythms of sleep and wakefulness (9, 11-14). Feeding behavior requires integration through eCB activation of $\mathrm{CB}_{1} \mathrm{R}$ at GABAergic synapses onto both LTS $^{+}$and LTS- DMH neurons (22). Our electrophysiological study with GAD67-GFP and vGLUT2iCreERT2 mutant mice revealed LTS $^{+}$and LTS $^{-}$neurons as GABAergic and glutamatergic neurons, respectively. Our electron microscopic study further suggests the existence of $\mathrm{CB}_{1} \mathrm{R}$ in both inhibitory and excitatory synapses in the $\mathrm{DMH}$. Thus, $\mathrm{DMH} \mathrm{CB}_{1} \mathrm{Rs}$ in both GABAergic and glutamatergic axon terminals synapsing with both GABAergic and glutamatergic DMH neurons are all likely targets of eCB. This idea is supported by induction of DSI from GABAergic and DSE from glutamatergic DMH neurons, as both DSI and DSE are mediated by eCB signaling $(17,25,26)$.

Surprisingly, isoflurane did not significantly affect DSI recorded from either GABAergic or glutamatergic DMH neurons, suggesting that general anesthetics do not significantly affect eCB signaling at GABAergic inputs onto $\mathrm{DMH}$ neurons, including the GABAergic SCN-DMH projection. Therefore, arousal from anesthesia is unlikely to require decreased eCB signaling at GABA- 
ergic inputs onto $\mathrm{DMH}$ neurons. Indeed, intra-DMH injection of the GABAR antagonist bicuculline did not significantly affect systemic AM281-accelerated arousal. The insignificant contributions of eCB signaling at DMH GABAergic synapses, including the GABAergic SCN-DMH projection, to arousal is further supported by our observation that an inducible deletion of $C B 1 R$ selectively from forebrain GABAergic neurons in GAD65-CB $\mathrm{R}-\mathrm{KO}$ mice did not significantly affect arousal.

In contrast to DSI, DSE recorded from both GABAergic and glutamatergic DMH neurons was significantly enhanced by isoflurane, strongly suggesting that anesthetics enhance eCB signaling at DMH glutamatergic synapses, although the mechanisms underlying isoflurane's increase of DSE, but not DSI, are unknown. Notably, intra-DMH injection of the NMDAR antagonist MK801 blocked systemic AM281-accelerated arousal, suggesting that decreased eCB signaling in the DMH during arousal from anesthesia occurs in glutamatergic inputs into the DMH. Because the $\mathrm{DMH}$ receives strong glutamatergic innervation from the prelimbic and infralimbic areas of the PFC (14), we hypothesized that decreased eCB signaling during arousal occurs at axon terminals of glutamatergic PFC-DMH projection. Indeed, we observed that a selective deletion of glutamatergic $C B 1 R$ either from the entire cerebral cortex or from the PFC mimicked the effects of systemic and intra-DMH application of AM281. Because decreased eCB signaling at axon terminals of glutamatergic $\mathrm{PFC}-\mathrm{DMH}$ projection could increase release of glutamate, inactivation or activation of PFC-DMH projection, respectively, would counteract or mimic the arousal-accelerating effects of AM281. This idea is supported by data showing that AM281's effects on arousal were counteracted by inactivation of neurons of the PFC-DMH projection and mimicked by activation of either somata or glutamatergic axon terminals of the PFC-DMH projection.

The DMH is the largest source of inputs to both the VLPO and Pef $(9,11,12)$. Approximately two-thirds of DMH-VLPO projections are GABAergic, and approximately two-thirds of DMH-Pef projections are glutamatergic (13). Our findings that intra-VLPO or intra-Pef injection of AM281 did not significantly affect arousal exclude a significant role of eCB signaling at either GABAergic VLPO or glutamatergic Pef synapses in arousal. This idea is further supported by the finding that arousal was not significantly affected by inducible deletion of $C B 1 R$ from either GABAergic neurons in GAD65-CB R-KO mice or hypothalamic glutamatergic neurons in vGLUT2-CB1R-KO mice. These results also suggest that the very low level of $\mathrm{CB}_{1} \mathrm{R}$ in somata of $\mathrm{DMH}$ neurons, as indicated by immunohistochemical staining (37), plays an insignificant role in arousal from anesthesia.

However, our findings that systemic AM281-accelerated arousal was abolished after either intra-VLPO injection of bicuculline or intra-Pef injection of MK801 suggest the association of GABAergic DMH-VLPO and glutamatergic DMH-Pef projections with arousal from anesthesia. This idea is also supported by that finding that inactivation of either DMH-VLPO or DMH-Pef projection counteracted systemic AM281-accelerated arousal. GABAergic neurons in the VLPO promote sleep through inhibition of the main ascending arousal nuclei in the brainstem, whereas orexinergic neurons in the Pef promote wakefulness via widespread excitation throughout the brain, including the brainstem ascend- ing arousal system $(1,9)$. We therefore propose that activation of the sleep-promoting inhibitory VLPO neurons and wake-promoting excitatory Pef neurons suppresses and promotes arousal, respectively. If activation of excitatory PFC-DMH projection participates in arousal from anesthesia, the accelerated arousal could result from enhancement of GABAergic DMH-VLPO projection, glutamatergic DMH-Pef projection, or both. This is because enhancement of inhibitory DMH-VLPO projection could inhibit the arousal-suppressing VLPO, hence accelerating arousal, which could also result from activation of the arousal-promoting Pef following enhancement of excitatory DMH-Pef projection. Indeed, we found that activation of DMH-VLPO projection or activation of either cell bodies or glutamatergic axon terminals of the DMH-Pef projection significantly accelerated arousal.

Our results clearly suggest that during the natural process of arousal after cessation of anesthesia, any driving force to promote arousal, such as enhancement of PFC-DMH, DMH-VLPO, or $\mathrm{DMH}$-Pef projection alone, is able to accelerate arousal. However, AM281-accelerated arousal may require a simultaneous enhancement of multiple brain circuitries, including PFC-DMH, DMH-VLPO, and DMH-Pef projections. Therefore, systemic AM281-accelerated arousal was abolished after systemic or intra-VLPO injection of bicuculline; after systemic or intra-Pef injection of MK801; or after chemogenetic-mediated inactivation of PFC-DMH, DMH-VLPO, or DMH-Pef projection. If activation of glutamatergic PFC-DMH, glutamatergic DMH-Pef, and GABAergic DMH-VLPO projections accelerates arousal, blockade or inactivation of each projection may delay arousal after anesthesia. Unexpectedly, we found recovery time after anesthesia was not significantly prolonged after systemic or intra-VLPO injection of bicuculline, after systemic or intra-Pef injection of MK801, or after chemogenetic-mediated inactivation of PFC-DMH, DMH-VLPO, or DMH-Pef projection. After the cessation of anesthesia, multiple mechanisms, such as the simultaneous involvement of multiple brain circuitries, may be necessary for arousal. Therefore, blockade or inactivation of the PFC-DMH-Pef/ VLPO circuitry alone may not strong enough to prolong arousal time after anesthesia. Accordingly, our finding that increased brain eCB signaling after a systemic injection of JZL195 prolonged recovery time indicates that the increased eCB signaling to prolong arousal may occur in multiple brain circuitries including the PFC-DMH-Pef/VLPO projection.

Here we employed a combination of behavioral, ultrastructural, electrophysiological, conditional mutagenesis, and chemogenetic strategies to investigate the questions of whether and how eCB signaling in PFC-DMH-VLPO/Pef circuitry modulates arousal. We provide evidence suggesting that after cessation of anesthesia, the driving force to increase eCB signaling disappears, leading to decreased $\mathrm{eCB}$ signaling at a specific hypothalamic node, i.e., the $\mathrm{DMH}$ glutamatergic synapses connecting the PFC-DMH, DMH-VLPO, and DMH-Pef circuitries. Decreased eCB signaling increases release of glutamate from axon terminals of the PFC-DMH projection, thus activating both inhibitory DMH-VLPO and excitatory DMH-Pef projections. Consequently, the arousal-suppressing VLPO is suppressed and the arousal-promoting Pef is activated, each or both of which accelerate arousal after anesthesia (Figure 8). 


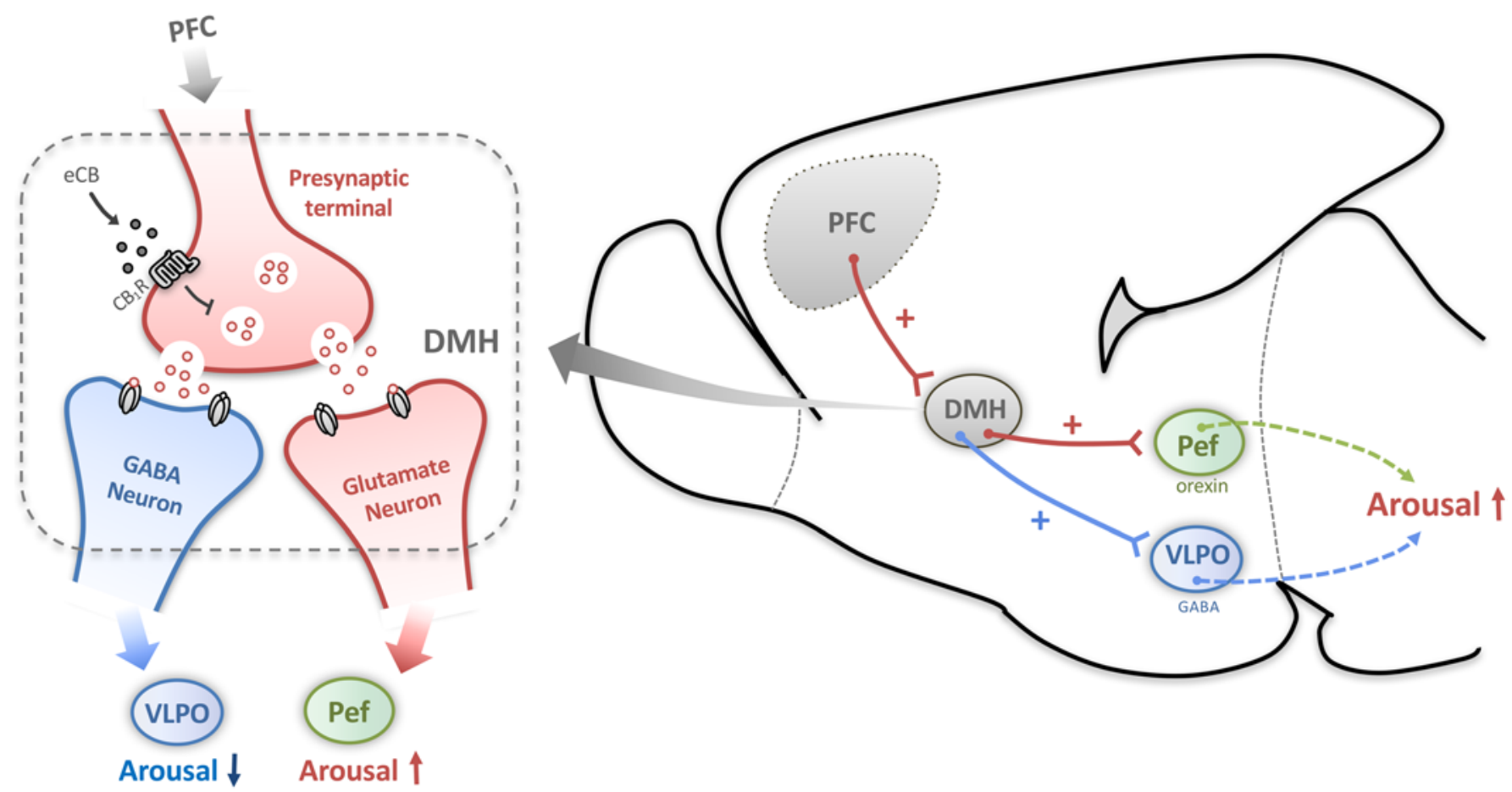

Figure 8. Proposed model for eCB modulation of brain circuitry participating in arousal from anesthesia. Glutamatergic neurons in the prelimbic and infralimbic areas of the PFC directly innervate both glutamatergic and GABAergic neurons in the DMH, which then send glutamatergic axons into the Pef and GABAergic axons into the VLPO, respectively. Glutamatergic DMH-Pef projection innervates excitatory orexin-containing neurons in the Pef, activation of which accelerates arousal. In contrast, GABAergic DMH-VLPO projection innervates inhibitory GABA-containing neurons in the VLPO, activation of which suppresses arousal. Thus, reduced eCB activation of $\mathrm{CB}_{1} \mathrm{R}$ in axon terminals of glutamatergic PFC-DMH projection increases presynaptic release of glutamate, which activates inhibitory DMH-VLPO projection, excitatory DMH-Pef projection, or both. Then suppression of the inhibitory VLPO neurons, enhancement of the excitatory Pef neurons, or both accelerate arousal after anesthesia.

In this study, we employed iCreERT2 mutant mice for electrophysiological, behavioral, and chemogenetic studies. The transgenic strategy has been used over the past couple decades for generation of traditional Cre mice, but it is now known that both the transgenic strategy and traditional Cre have potential problems. For example, the traditional bacteriophage-derived Cre is not optimal for eukaryotes due to its codon usage of prokaryotes, and its $65 \mathrm{CpG}$ dinucleotides may induce epigenetic silencing (38). To overcome these potential problems, we generated vGLUT1-iCreERT2, vGLUT2-iCreERT2, and GAD65-iCreERT2 mutant mice with a knockin of iCreERT2 $(38,39)$ into the Vglut1, Vglut2, or GAD65 locus. Mice received 8 daily injection of tamoxifen 1 week before use (40). In the case of intra-DMH injection of AAV-mCherry into vGLUT2-iCreERT2 mice, red fluorescent cells were detected in the $\mathrm{DMH}$, signifying the successful inversion and expression of the mCherry and EYFP by Cre. This finding was confirmed with in situ hybridization of Vglut1 or Vglut2 and iCre mRNAs in the PFC and DMH.

The present study relies heavily on a large number of experiments with intracerebral microinjections of various receptor agonists or antagonists, $\mathrm{AAV}$ vectors, or vehicle. In order to minimize the potential problem of acute reaction following intracerebral insertion of injection needle, we collected behavioral data either acutely after an intracerebral administration of various receptor agonists or antagonists or vehicle through the guide cannula implanted 1-2 weeks in advance, or 1-2 weeks after intracere- bral application of AAV vectors or vehicle. To achieve the goal of restricting the treatment within the boundaries of the VLPO, Pef, $\mathrm{DMH}$, or PFC, a well-trained experimenter steadily injected a 0.3 $\mu \mathrm{l}$ volume in 5 minutes. We excluded all animals with an injection site outside the target brain region. Although we cannot exclude the possibility that a small amount of injected agent spread outside the target brain region, the major effects were likely achieved through the effective action of injected agents within the target brain region. This idea is supported by our finding that the same amount of AM281 produced significant behavioral effects after intra-DMH injection but not after intra-Pef injection, even though these two hypothalamic regions are adjacent (Supplemental Figure 4).

\section{Methods}

Animals. Behavioral and electrophysiological studies employed male Sprague-Dawley rats (Charles River) weighing 250-300 g and 75-100 g, respectively. Electron microscopy studies used adult male C57BL/6 mice (Charles River) weighing 25-30 g. Rats and mice were housed in groups of 2 and 4, respectively, in standard cages under standard laboratory conditions (12-hour light/12-hour dark cycle, $22 \pm 2^{\circ} \mathrm{C}$, food and water ad libitum). Animals were allowed to adapt to laboratory conditions for at least 1 week and adapt to the testing room for at least 30 minutes before behavioral tests.

Generation of mutant mice. CB1R-floxed mice were produced as described previously (29). vGLUT1-iCreERT2, vGLUT2-iCreERT2, and GAD65-iCreERT2 mutant mice were generated by Biocytogen 
with the same procedures. Briefly, to make the targeting construct, the appropriate regions were designed and cloned from BAC library to be the homologous arms. For vGLUT1 targeting construct, the $5.8-\mathrm{kb}$ of genomic fragment from the upstream of exon 2 to the downstream of exon 11 and the 6.7-kb fragment beginning from the $3^{\prime}$ UTR of the Vglut1 gene (also known as Slc17a7) were cloned. For vGLUT2 targeting construct, the $5^{\prime}$ homologous region is a 9.0kb fragment containing Vglut2 exons 9, 10, and 11 and the intron sequences, and the $3^{\prime}$ homologous region is a $4.9-\mathrm{kb}$ one containing the Vglut2 $3^{\prime}$ UTR. For GAD65-iCreERT2 mice, the $5^{\prime}$ and $3^{\prime}$ homologous regions are an 8.5-kb fragment spanning exons 13,14 , and 15 , and a $4.0-\mathrm{kb}$ fragment beginning from the $3^{\prime}$ UTR of GAD65 gene, respectively. To avoid disruption of normal gene expression, $2 A$-CreERT2 was introduced between the coding sequence of the last exon of each gene and its $3^{\prime}$ UTR. The final targeting vector included the $5^{\prime}$ homologous region, a neomycin phosphotransferase expression (Neo) cassette with two FRT sites flanked, the coding sequence of the last exon, $2 A$-CreERT2, and the $3^{\prime}$ homologous region. To generate the knockin mice, the linearized targeting construct obtained with the restriction endonuclease indicated in Supplemental Figures 5, 8, and 11 was electroporated into embryonic stem (ES) cells (Biocytogen) with a C57BL/6 background. Random integrations will be negatively selected by diphtheria toxin A (DTA). Positive clones were screened by G418 and then identified by Southern blot analysis. The targeted clones were then injected into C57BL/6 blastocysts. Chimeric mice were generated and bred with WT mice. The primers used to identify each type of knockin mice are shown in Supplemental Table 1. The CB1R-floxed mice were crossed with the vGLUT1-iCreERT2, vGLUT2-iCreERT2, or GAD65-iCreERT2 mutant mice to generate vGLUT1-CB $\mathrm{R}-\mathrm{KO}$, vGLUT2-CB ${ }_{1} \mathrm{R}-\mathrm{KO}$, and $\mathrm{GAD} 65-\mathrm{CB}_{1} \mathrm{R}-\mathrm{KO}$ mouse lines with the same protocol described previously (29). These 3 lines of mice and all iCreERT2 mice received 8 daily injections of tamoxifen (1 mg/ mouse/d; dissolved in corn oil) 1 week before use (40).

FISH. The pBluescript II KS (+) plasmids containing complementary DNA fragments of either Vglut1 (nucleotides 855-1,788; GenBank XM_133432.2) or Vglut2 (nucleotides 848-2,044; GenBank NM_080853.2) were a gift from Minhua Zheng (Fourth Military Medical University, Xi'an, China) and are described elsewhere (41). The pBBI plasmid (Addgene, 65795) containing iCre coding sequences (CDs) was subcloned to reverse the iCre CDs to obtain pBBI-reversed plasmid with the complementary DNA fragment of iCre (nucleotides 1-1,053) under the T7 promoter. Using the linearized plasmids as templates, we synthesized sense and antisense single-strand RNA probes with a fluorescein labeling kit (Invitrogen, MP 32956). Double FISH on cryosections was carried out as previously described $(41,42)$. In short, $20-\mu \mathrm{m}$ coronal brain sections were mounted on microscope slides (Fisher Scientific, J1800AMNZ). After fixation with $4 \%$ paraformaldehyde (PFA), the slides were treated with $10 \mu \mathrm{g} / \mathrm{ml}$ proteinase $\mathrm{K}$ (Invitrogen, AM2546) in buffer containing $5 \mathrm{mM}$ EDTA and $50 \mathrm{mM}$ Tris- $\mathrm{HCl}, \mathrm{pH} 7.5$, for 15 minutes at room temperature and post-fixed with $4 \%$ PFA for 15 minutes. After prehybridization, the sections were incubated for 16 hours at $62^{\circ} \mathrm{C}$ in hybridization buffer with a mixture of $1 \mu \mathrm{g} / \mathrm{ml}$ Alexa Fluor 488-labeled probe for vGLUT1 or vGLUT2 and $1 \mu \mathrm{g} / \mathrm{ml}$ Alexa Fluor 594-labeled probe for iCre. The hybridization buffer contained $50 \%$ (vol/vol) deionized formamide (Sigma-Aldrich, F9037), $200 \mathrm{mM} \mathrm{NaCl}, 5 \mathrm{mM}$ EDTA, 10 mM Tris- $\mathrm{HCl} \mathrm{pH}$ 7.5, 5 mM
$\mathrm{NaH}_{2} \mathrm{PO}_{4}, 5 \mathrm{mM} \mathrm{Na} \mathrm{HPO}_{4}, 0.01 \mathrm{mg} / \mathrm{ml}$ yeast tRNA (Sigma-Aldrich, TRNABAK-RO), $1 \times$ Denhardt's solution (Sigma-Aldrich, D2532), and $10 \% \mathrm{wt} / \mathrm{vol}$ dextran sulfate (Sigma-Aldrich, D8906). After washes with a series of saline sodium citrate (SSC) solutions, the sections were counterstained with $1 \mu \mathrm{g} / \mathrm{ml}$ DAPI (Thermo Fisher Scientific, D1306) for 10 minutes and were coverslipped with SlowFade Gold antifade reagent (Invitrogen, MP 32956). Photographs were taken with an Olympus BX51 microscope or Zeiss confocal microscope. No hybridization signal was detected when sense RNA probes were used. The distribution of Vglut1 and Vglut2 mRNA in the mouse brain was compared with that reported in a recent study (43).

Quantification of recovery time from anesthesia. Five minutes before anesthesia, an animal was placed into a rotating Plexiglas anesthesia chamber (Supplemental Figure 2) (20) connected to an isoflurane or sevoflurane vaporizer (Ohmeda). Anesthesia was $1.4 \%$ isoflurane or $2.4 \%$ sevoflurane in $100 \%$ oxygen, which was continuously delivered into the chamber at $1.5 \mathrm{l} / \mathrm{min}$ for 30 minutes. Immediately after cessation of anesthesia, pure oxygen was continuously delivered at $1.5 \mathrm{l} /$ min into the chamber, where the rat was placed in a dorsal recumbent position on the floor of the chamber for quantification of RORR or recovery time, i.e., the time from cessation of isoflurane administration to resumption of a prone position.

EEG recording. Rats were anesthetized with $1.5 \%-2 \%$ isoflurane ( $\mathrm{vol} / \mathrm{vol})$ and placed in a stereotaxic frame. Body temperature was kept constant throughout the operation by using a heating pad. After anesthesia was established, the skin was incised to expose the skull and the overlying connective tissue removed. Two pairs of stainless steel screws were implanted bilaterally over the frontal cortex (anterior-posterior [AP] 3.9, medial-lateral [ML] $\pm 2 \mathrm{~mm}$ ) and occipital cortex (AP-7.4 mm, ML $\pm 5 \mathrm{~mm}$ ) (44). The screws and electrodes were secured to the skull using dental cement. After surgery, rats were allowed to recover for at least 1 week before experiments. Rats were acclimated to the EEG recoding cable for 30 minutes, during which EEG was recorded with a sampling frequency of 0-150 Hz using Powerlab 16/35 amplifier system (PL3516, ADInstruments) and Labchart Pro v8.0.10 (MLU60/8, ADInstruments). Then the rats were transferred into the Plexiglas anesthesia chamber for isoflurane anesthesia for $30 \mathrm{~min}$ utes as described above. Vehicle for AM281 was injected 15 minutes before the end of anesthesia. EEG was recorded continuously until 15 minutes after RORR. EEG spectrum analysis and spectrograms were performed by using MATLAB software (Mathworks).

Chemical/viral treatment. For consciousness recovery study, animals received an i.p. injection or an intra-hypothalamic (i.h.) injection of a chemical, virus vector, or vehicle 15 minutes before the cessation of isoflurane anesthesia, with or without i.p. or i.h. administration of another chemical or vehicle 25 minutes before anesthesia cessation. The following chemicals were purchased from Tocris Bioscience and dissolved in physiological saline, unless otherwise stated: AM281 (0.3, 1.5, or $3 \mathrm{mg} / \mathrm{kg}$, i.p.; $0.1 \mu \mathrm{g} / 0.3 \mu \mathrm{l} /$ side, i.h.; dissolved in 5\% Tween-80, 5\% DMSO, and 90\% physiological saline); NESSO327 (Cayman Chemical; $0.3 \mathrm{mg} / \mathrm{kg}$, i.p.; dissolved in 5\% Tween-80, 5\% DMSO, and 90\% physiological saline) (21); MK801 (0.02 or $0.2 \mathrm{mg} /$ $\mathrm{kg}$, i.p.; 0.02 or $0.005 \mu \mathrm{g} / 0.3 \mu \mathrm{l} / \mathrm{side}$, i.h.); bicuculline (0.5 or $2 \mathrm{mg} /$ kg, i.p.; 0.2, 0.4, or $4 \mu \mathrm{g} / 0.3 \mu \mathrm{l} / \mathrm{side}$, i.h.); JZL195 (20 mg/kg, i.p.; dissolved in 5\% Tween-80, 5\% DMSO, and 90\% physiological saline). All AAV viruses, including AAV8-hSyn-DIO-mCherry (AAV-mCherry), AAV8-hSyn-DIO-hM4Di-mCherry (AAV-hM4Di-mCherry), and 
AAV8-hSyn-DIO-hM3Dq-mCherry (AAV-hM3Dq-mCherry), were purchased from the University of North Carolina Gene Therapy Center, Vector Core. Aliquots of virus vectors were stored at $-80^{\circ} \mathrm{C}$ before an i.h. microinjection. PRV-Cre stocks were prepared and titered as described previously (45), except that each $10-\mathrm{cm}$ dish of virus stock was resuspended in $500 \mu \mathrm{l}$ and stored in $50-\mu \mathrm{l}$ aliquots at $-80^{\circ} \mathrm{C}$. On the day of surgery, stocks were thawed, cell debris was pelleted by centrifugation at high speed for 1 minute in a benchtop centrifuge, and the supernatant was carefully taken for injection.

Under isoflurane anesthesia, two guide cannulae (33-gauge, Plastics One) were implanted into the bilateral DMH (AP -3.2, ML \pm 0.75 , dorsal-ventral [DV] $-8.8 \mathrm{~mm}$ ), VLPO (AP $-0.4, \mathrm{ML} \pm 1, \mathrm{DV}-9$ $\mathrm{mm})$, Pef (AP -3.1, ML $\pm 1.4, \mathrm{DV}-8.6 \mathrm{~mm}$ ), or PFC (AP +3.7, ML \pm 0.7 , $\mathrm{DV}-4.8 \mathrm{~mm})$, after which the cannulae were affixed to the skull with screws and dental acrylic and then filled with stylets. One week later, animals received isoflurane anesthesia for quantification of recovery time, during which an i.h. injection of $0.3 \mu \mathrm{l}$ of the chemical or vehicle was performed in a 5-minute period. For chemogenetic study, rats were anesthetized to receive an i.h. injection of $0.5 \mu \mathrm{l}$ PR-Cre $\left(6.0 \times 10^{5}\right.$ to $7.0 \times 10^{5}$ genome copies per ml $)$, AAV-hM4Di-mCherry or AAV-hM3Dq-mCherry $\left(4.6 \times 10^{12}\right.$ genome copies per ml) $(46)$, or AAV-mCherry into bilateral DMH, VLPO, Pef, or PFC at a speed of $0.1 \mu \mathrm{l} / \mathrm{min}$. Seven or 14 days after surgery, rats were given CNO (Cayman Chemical; $5 \mathrm{mg} / \mathrm{kg}$, i.p.; dissolved in DMSO), followed 1 hour later by isoflurane anesthesia for quantification of recovery time. Finally, all rats were perfused transcardially with $4 \%$ PFA. Brains were sectioned to identify cannula placement and/or the location of mCherry fluorescence expression.

The vGLUT2-iCreERT2 and vGLUT1-iCreERT2 mice used for chemogenetic study of the activation of DMH-Pef or PFC-DMH terminals received bilateral intra-DMH injection (AP $-1.7, \mathrm{ML} \pm 0.5, \mathrm{DV}$ $-5.6 \mathrm{~mm}$ ) or intra-PFC injection ( $\mathrm{AP}+1.7, \mathrm{ML} \pm 0.4, \mathrm{DV}-3.35 \mathrm{~mm}$ ) of AAV-hM3Dq-mCherry and implantation of cannulae into the Pef (AP $-1.9, \mathrm{ML} \pm 1.0, \mathrm{DV}-4.8 \mathrm{~mm}$ ) or DMH (AP $-1.7, \mathrm{ML} \pm 0.5, \mathrm{DV}-4.6 \mathrm{~mm}$ ) as described above. Two weeks after surgery, vGLUT2-iCreERT2 mice received intra-Pef administration of CNO $(10 \mu \mathrm{M} / 0.2 \mu \mathrm{l} / \mathrm{side})$ and vGLUT1-iCreERT2 mice received intra-DMH administration of CNO 4 hours before experiments to study consciousness recovery after anesthesia.

The $C B 1 R$-floxed mice used for AAV-mediated deletion of $C B 1 R$ in $\mathrm{PFC}$ glutamatergic neurons received bilateral intra-PFC injection (AP $+1.7, \mathrm{ML} \pm 0.4, \mathrm{DV}-3.35 \mathrm{~mm}$ ) of AAV-CaMKII-iCre-GFP (a gift from Liping Wang, Shenzhen Institutes of Advanced Technology, Chinese Academy of Sciences, Shenzhen, China) and 6 weeks later underwent electron microscopic study or behavioral study to quantify the recovery time after isoflurane anesthesia.

Electron microscopy analysis. See our recent study (29) for detailed protocols. Briefly, animals were transcardially perfused under anesthesia with $100 \mathrm{ml}$ of $0.1 \%$ glutaraldehyde, $4 \%$ formaldehyde, and $15 \%$ saturated picric acid in phosphate buffer ( $\mathrm{PB}, \mathrm{pH}$ 7.4). Coronal hypothalamus vibrosections were cut at $50 \mu \mathrm{m}$. After being freezethawed with liquid nitrogen, the sections were incubated for 1 hour in 0.05 M Tris-HCl-buffered saline (TBS; pH 7.4) containing 20\% (vol/ vol) normal goat serum, for 24 hours in primary polyclonal rabbit anti-CB R (1:100) antibody (47) with or without monoclonal mouse anti-vGlUT1 antibody (1:500, catalog 135311, Synaptic Systems) or polyclonal guinea pig anti-vGLUT2 antibody (1:200, catalog 135404,
Synaptic Systems). After several washes in TBS, tissue sections were incubated overnight with a mixture of 1.4-nm gold particle-conjugated goat ant-rabbit IgG (1:100, Nanoprobes) with or without biotinylated donkey anti-mouse IgG (1:200, Millipore) or biotinylated donkey anti-guinea pig IgG (1:200, Millipore). Next, the sections were postfixed with $1 \%$ (wt/vol) glutaraldehyde in $0.1 \mathrm{M} \mathrm{PB}$ ( $\mathrm{pH}$ 7.4) for 10 minutes and washed in distilled water; this was followed by silver enhancement in the dark with an HQSilver Kit (Nanoprobes). For double labeling, sections were incubated in avidin-biotin-peroxidase complex (1:50, Elite ABC Kit; Vector Laboratories) for 6 hours and then in $0.02 \% 3,3^{\prime}$-diaminobenzidine- $4 \mathrm{HCl}$ and $0.003 \%$ (vol/vol) $\mathrm{H}_{2} \mathrm{O}_{2}$ for 20-30 minutes. Then all the sections were placed in $0.1 \mathrm{M} \mathrm{PB}$ containing $1 \% \mathrm{OsO}_{4}$ for 1 hour and finally counterstained with $1 \%$ uranyl acetate in $70 \%$ ethanol for 1 hour. After dehydration, the sections were mounted on silicon-coated glass slides and flat embedded in epoxy resin (Durcupan; Fluka, Buchs). Tissues containing the DMH or the Pef were cut on an ultramicrotome into 60-nm-thick sections, which were mounted on single-slot grids membrane and examined with a JEM-1400 electron microscope. $\mathrm{CB}_{1}$ R-positive labeling was considered if at least 3 immunoparticles were within approximately $30 \mathrm{~nm}$ from the membrane.

Electrophysiology analysis. After decapitation, the brain was removed, and coronal hypothalamic vibrosections $(300 \mu \mathrm{m})$ were cut and maintained in the artificial cerebral spinal fluid (ACSF) (in mM: 125 $\mathrm{NaCl}, 25 \mathrm{NaHCO}_{3}, 1.25 \mathrm{KCl}, 1.25 \mathrm{KH}_{2} \mathrm{PO}_{4}, 1.5 \mathrm{MgCl}_{2}, 1 \mathrm{CaCl}_{2}$, and 16 glucose) saturated with $95 \% \mathrm{O}_{2} / 5 \% \mathrm{CO}_{2}$. The patch pipettes contained an intracellular solution (in mM: $120 \mathrm{~K}$ gluconate, $20 \mathrm{KCl}, 10 \mathrm{HEPES}$, 10 phosphocreatine Na salt, 2 ATP Na salt, 0.4 GTP Na salt, $2 \mathrm{MgCl}_{2}$, $\mathrm{pH}$ 7.35). To record AMPAR-mediated EPSCs, $50 \mu \mathrm{M}$ D-APV and $10 \mu \mathrm{M}$ BMI were added in the perfusion solution to block NMDAR-mediated EPSCs and GABAR-mediated inhibitory postsynaptic currents (IPSCs), respectively. To record IPSCs, $10 \mu \mathrm{M}$ CNQX was added in the perfusion solution to block AMPAR-mediated EPSCs. To record postsynaptic currents (PSCs), neurons were whole-cell voltage clamped at $-70 \mathrm{mV}$ with a Multiclamp 700B amplifier (Molecular Devices). To evoke PSCs, a stable whole-cell recording configuration was established on the DMH cell. Then a second stimulation glass electrode (2-6 M $\Omega$ ) filled with $2 \mathrm{M} \mathrm{NaCl}$ was lowered into the slice $100 \mu \mathrm{m}$ away from the recording site. PSCs were collected by voltage clamp recordings using a stimulus pulse of a single square wave (stimulation intensity: $100 \mu \mathrm{s}, 50-200$ $\mu \mathrm{A})$. The data were acquired with pCLAMP 10.4 (Molecular Devices) at a sampling rate of 5-10 kHz, measured, and plotted with pCLAMP 10.0 and GraphPad Prism 6.0. To evoke DSI/DSE at 4-second intervals, cells were depolarized from -70 to $0 \mathrm{mV}$ for 5 seconds. Saturated isoflurane was prepared by mixing isoflurane and ACSF in a tightly sealed glass bottle and then shaking overnight to reach the saturated concentration of 13-15 mM. After dilution to 1:10 (1.3-1.5 mM) with ACSF, the isoflurane solution was delivered with polytetrafluorethylene tubing to the recording chamber with the final isoflurane concentration at clinically relevant 200-300 $\mu \mathrm{M}(48-50)$. Sevoflurane-containing gas mixtures $(2 \%)$ were prepared by delivering the carbogen $\left(95 \% \mathrm{O}_{2} / 5 \% \mathrm{CO}_{2}\right)$ through a calibrated vaporizer. As determined in a recent study (51), the concentration of sevoflurane in the ACSF bubbled with the gas mixtures for $>30$ minutes was $200 \mu \mathrm{M}$, which is the value within the range for clinical and in vivo experimental use $(52,53)$. High-quality polytetrafluorethylene tubing and valves were used to minimize the loss of sevoflurane. To test whether the effects of isoflurane or sevoflurane were 
reversible, isoflurane or sevoflurane solution was replaced with normal $\mathrm{ACSF}$ at 4 minutes after application. To test whether isoflurane inhibition of firing rate was $\mathrm{CB}_{1} \mathrm{R}$ dependent, we applied ACSF solution containing AM281 $(1 \mu \mathrm{M})$ onto brain slices, and AM281 solution was present throughout the whole experiment. To identify DMH glutamatergic neurons in the vGLUT2-iCreERT2 mice, young mice received a microinjection of AAV-mCherry into the $\mathrm{DMH}$ at a rate of $0.15 \mu \mathrm{l} / \mathrm{min}$ for 2 minutes; 1 week later hypothalamic slices were prepared as described above. To reveal chemogenetic functioning, young rats received a microinjection of AAV-hM3Dq-mCherry into the DMH (AP -3.0, ML $\pm 0.6, \mathrm{DV}-8.9 \mathrm{~mm}$ ) and PRV-Cre into the VLPO (AP -0.3, ML \pm 0.9 , $\mathrm{DV}-8.1 \mathrm{~mm}$ ) at a rate of $0.15 \mu \mathrm{l} / \mathrm{min}$ for 2 minutes. One week later, rats were killed for preparation of hypothalamic slices as described above, and mCherry-positive DMH neurons were identified and recorded. After acquisition of stable recordings for 5-7 minutes, slices were perfused with ACSF solution containing $1 \mu \mathrm{M} \mathrm{CNO}$. Changes in membrane potential and spike activity were monitored, recorded, measured, and plotted with a pCLAMP 10.0 and GraphPad Prism 6.0. To reveal terminal release properties of DMH-Pef projection in vGLUT2-iCreERT2 mice, a stable whole-cell recording configuration was established on a Pef neuron, and a second stimulation glass electrode (2-6 M 2 ) filled with $2 \mathrm{M} \mathrm{NaCl}$ was placed about $100 \mu \mathrm{m}$ away from the patched neuron. Paired pulse facilitation (PPF) or depression (PPD) was collected by voltage clamp recordings using a stimulus pulse with a single square wave every 10 seconds. Paired-pulse responses were performed at intervals of $100 \mathrm{~ms}$. PPR was measured as the amplitude ratio of the second to the first postsynaptic response from an average of 10 pairs of pulses. The data were acquired with pCLAMP 10.4 (Molecular Devices), and measured and plotted with Clampfit 10.4 and GraphPad Prism 6.0.

Statistics. Results are reported as mean \pm SEM. Statistical analysis of the data was performed using 2-tailed Student's $t$ test, 1-way ANOVA, followed by Tukey's post-hoc test. Statistical significance was set at $P<0.05$.
Study approval. The present studies in animals were reviewed and approved (ACC-2012-004) by the Animal Care Committee of the University of Ottawa Institute of Mental Health Research at the Royal, Ottawa. Similar procedures were also approved by the Animal Care Committee of the Fourth Military Medical University, Xi'an.

\section{Author contributions}

HZ, LT, NG, FG, YL, RX, XL, JL, RB, LEP, FW, CXL, YR, XW, and JL collected the data and performed the analyses. HZ, LT, NG, FG, RX, RB, LEP, KM, SXW, ZX, LX, JL, HD, LX, and XZ wrote the manuscript. XZ, LX, and HD supervised the project.

\section{Acknowledgments}

This project was supported by funding from the Canadian Institutes of Health Research (CIHR) to XZ (MOP123249, MOP123256); from the National Natural Science Foundation of China to HD (grant 81371510), JL (grant 81571074), and HZ (grant 81403464); from the International Cooperation and Exchange of the National Natural Science Foundation of China to LX (grant 81420108013); an equipment grant from the Canadian Foundation for Innovation to XZ; and NIH grants (DA011322 and DA021696) to KM. LEP acknowledges support from Jeffrey M. Friedman and the JPB Foundation.

Address correspondence to: Xia Zhang, University of Ottawa Institute of Mental Health Research at the Royal, 1145 Carling Avenue, Ottawa, Ontario K1Z 7K4, Canada. Phone: 613.722.6521 ext 6218; E-mail:Xia.Zhang@theroyal.ca. Or to: Lize Xiong or Hailong Dong, Department of Anesthesiology and Perioperative Medicine, Xijing Hospital, Fourth Military Medical University, Number 169 Changle West Road, Xi'an, Shaanxi Province 710032, China. Phone: 86.0298.477.5012; E-mail: mzkxlz@126.com (L. Xiong); Phone: 86.0298.477.5337; E-mail: hldong6@hotmail.com (H. Dong).
1. Franks NP. General anaesthesia: from molecular targets to neuronal pathways of sleep and arousal. Nat Rev Neurosci. 2008;9(5):370-386.

2. Alkire MT, Hudetz AG, Tononi G. Consciousness and anesthesia. Science. 2008;322(5903):876-880.

3. Hameroff SR. The entwined mysteries of anesthesia and consciousness: is there a common underlying mechanism? Anesthesiology. 2006;105(2):400-412.

4. Leung LS, Luo T, Ma J, Herrick I. Brain areas that influence general anesthesia. Prog Neurobiol. 2014;122:24-44

5. Lydic R, Baghdoyan HA. Sleep, anesthesiology, and the neurobiology of arousal state control. Anesthesiology. 2005;103(6):1268-1295.

6. Scharf MT, Kelz MB. Sleep and anesthesia interactions: a pharmacological appraisal. Curr Anesthesiol Rep. 2013;3(1):1-9.

7. Weiser TG, et al. Estimate of the global volume of surgery in 2012: an assessment supporting improved health outcomes. Lancet. 2015; 385(Suppl 2):S11.

8. Franks NP, Zecharia AY. Sleep and general anesthesia. Can J Anaesth. 2011;58(2):139-148.

9. Saper CB, Scammell TE, Lu J. Hypothalamic regulation of sleep and circadian rhythms. Nature. 2005;437(7063):1257-1263.
10. Aston-Jones G, Chen S, Zhu Y, Oshinsky ML. A neural circuit for circadian regulation of arousal. Nat Neurosci. 2001;4(7):732-738.

11. Deurveilher S, Burns J, Semba K. Indirect projections from the suprachiasmatic nucleus to the ventrolateral preoptic nucleus: a dual tract-tracing study in rat. Eur J Neurosci. 2002;16(7):1195-1213.

12. Deurveilher S, Semba K. Indirect projections from the suprachiasmatic nucleus to major arousal-promoting cell groups in rat: implications for the circadian control of behavioural state. Neuroscience. 2005;130(1):165-183.

13. Chou TC, Scammell TE, Gooley JJ, Gaus SE, Saper CB, Lu J. Critical role of dorsomedial hypothalamic nucleus in a wide range of behavioral circadian rhythms. J Neurosci. 2003;23(33):10691-10702.

14. Myers B, Mark Dolgas C, Kasckow J, Cullinan WE, Herman JP. Central stress-integrative circuits: forebrain glutamatergic and GABAergic projections to the dorsomedial hypothalamus, medial preoptic area, and bed nucleus of the stria terminalis. Brain Struct Funct. 2014;219(4):1287-1303.

15. Blankman JL, Cravatt BF. Chemical probes of endocannabinoid metabolism. Pharmacol Rev. 2013;65(2):849-871.

16. Baker D, Pryce G, Giovannoni G, Thompson AJ.
The therapeutic potential of cannabis. Lancet Neurol. 2003;2(5):291-298.

17. Chevaleyre V, Takahashi KA, Castillo PE. Endocannabinoid-mediated synaptic plasticity in the CNS. Annu Rev Neurosci. 2006;29:37-76.

18. Katona I, Freund TF. Multiple functions of endocannabinoid signaling in the brain. Annu Rev Neurosci. 2012;35:529-558.

19. Patel S, et al. The general anesthetic propofol increases brain $\mathrm{N}$-arachidonylethanolamine (anandamide) content and inhibits fatty acid amide hydrolase. Br J Pharmacol. 2003;139(5):1005-1013.

20. Baker R, et al. Altered activity in the central medial thalamus precedes changes in the neocortex during transitions into both sleep and propofol anesthesia. J Neurosci. 2014;34(40):13326-13335.

21. Meye FJ, Trezza V, Vanderschuren LJ, Ramakers GM, Adan RA. Neutral antagonism at the cannabinoid 1 receptor: a safer treatment for obesity. Mol Psychiatry. 2013;18(12):1294-1301.

22. Crosby KM, Inoue W, Pittman QJ, Bains JS. Endocannabinoids gate state-dependent plasticity of synaptic inhibition in feeding circuits. Neuron. 2011;71(3):529-541.

23. Tamamaki N, Yanagawa Y, Tomioka R, Miyazaki J, Obata K, Kaneko T. Green fluorescent 
protein expression and colocalization with calretinin, parvalbumin, and somatostatin in the GAD67-GFP knock-in mouse. J Comp Neurol. 2003;467(1):60-79.

24. Wittmann G, et al. Distribution of type 1 cannabinoid receptor (CB1)-immunoreactive axons in the mouse hypothalamus. J Comp Neurol. 2007;503(2):270-279.

25. Straiker A, Mackie K. Depolarization-induced suppression of excitation in murine autaptic hippocampal neurones. J Physiol (Lond). 2005;569(Pt 2):501-517.

26. Yoshida $\mathrm{T}$, et al. Unique inhibitory synapse with particularly rich endocannabinoid signaling machinery on pyramidal neurons in basal amygdaloid nucleus. Proc Natl Acad Sci U S A. 2011;108(7):3059-3064.

27. Fujiyama F, Furuta T, Kaneko T. Immunocytochemical localization of candidates for vesicular glutamate transporters in the rat cerebral cortex. J Comp Neurol. 2001;435(3):379-387.

28. Wang Y, et al. Monoacylglycerol lipase inhibitors produce pro- or antidepressant responses via hippocampal CA1 GABAergic synapses. Mol Psychiatry. 2017;22(2):215-226.

29. Alexander GM, et al. Remote control of neuronal activity in transgenic mice expressing evolved G protein-coupled receptors. Neuron. 2009;63(1):27-39.

30. Armbruster BN, Li X, Pausch MH, Herlitze S, Roth BL. Evolving the lock to fit the key to create a family of $G$ protein-coupled receptors potently activated by an inert ligand. Proc Natl Acad Sci U S A. 2007;104(12):5163-5168.

31. Ferguson SM, et al. Transient neuronal inhibition reveals opposing roles of indirect and direct pathways in sensitization. Nat Neurosci. 2011;14(1):22-24.

32. Atasoy D, Aponte Y, Su HH, Sternson SM. A FLEX switch targets Channelrhodopsin-2 to multiple cell types for imaging and long-range circuit mapping. J Neurosci. 2008;28(28):7025-7030.

33. Chaudhury D, et al. Rapid regulation of depression-related behaviours by control of midbrain dopamine neurons. Nature. 2013;493(7433):532-536.

34. Walsh JJ, et al. Stress and CRF gate neural activation of BDNF in the mesolimbic reward pathway. Nat Neurosci. 2014;17(1):27-29.

35. Stachniak TJ, Ghosh A, Sternson SM. Chemogenetic synaptic silencing of neural circuits localizes a hypothalamus $\rightarrow$ midbrain pathway for feeding behavior. Neuron. 2014;82(4):797-808.

36. Zhu H, Pleil KE, Urban DJ, Moy SS, Kash TL, Roth BL. Chemogenetic inactivation of ventral hippocampal glutamatergic neurons disrupts consolidation of contextual fear memory. Neuropsychopharmacology. 2014;39(8):1880-1892.

37. Hrabovszky E, Wittmann G, Kalló I, Füzesi T, Fekete C, Liposits Z. Distribution of type 1 cannabinoid receptor-expressing neurons in the septal-hypothalamic region of the mouse: colocalization with GABAergic and glutamatergic markers. J Comp Neurol. 2012;520(5):1005-1020.

38. Shimshek DR, et al. Codon-improved Cre recombinase (iCre) expression in the mouse. Genesis. 2002;32(1):19-26.

39. Cox BC, Liu Z, Lagarde MM, Zuo J. Conditional gene expression in the mouse inner ear using CreloxP. JAssoc Res Otolaryngol. 2012;13(3):295-322.

40. Hirrlinger PG, Scheller A, Braun C, Hirrlinger J, Kirchhoff F. Temporal control of gene recombination in astrocytes by transgenic expression of the tamoxifen-inducible DNA recombinase variant CreERT2. Glia. 2006;54(1):11-20.

41. Nakamura K, et al. Transiently increased colocalization of vesicular glutamate transporters 1 and 2 at single axon terminals during postnatal development of mouse neocortex: a quantitative analysis with correlation coefficient. Eur JNeurosci. 2007;26(11):3054-3067.

42. Jolly S, Fudge A, Pringle N, Richardson WD, $\mathrm{Li} \mathrm{H.} \mathrm{Combining} \mathrm{double} \mathrm{fluorescence} \mathrm{in}$ situ hybridization with immunolabelling for detection of the expression of three genes in mouse brain sections. J Vis Exp. 2016;109(109):e53976.
43. Herzog E, et al. The existence of a second vesicular glutamate transporter specifies subpopulations of glutamatergic neurons. J Neurosci. 2001;21(22):RC181.

44. Dong $\mathrm{H}$, et al. Activation of orexin signal in basal forebrain facilitates the emergence from sevoflurane anesthesia in rat. Neuropeptides. 2009;43(3):179-185.

45. Card JP, Enquist LW. Transneuronal circuit analysis with pseudorabies viruses. Curr Protoc Neurosci. 2014;68:1.5.1-1.539.

46. Krashes MJ, et al. Rapid, reversible activation of AgRP neurons drives feeding behavior in mice. J Clin Invest. 2011;121(4):1424-1428.

47. Bodor AL, et al. Endocannabinoid signaling in rat somatosensory cortex: laminar differences and involvement of specific interneuron types. JNeurosci. 2005;25(29):6845-6856.

48. Liachenko S, Tang P, Somogyi GT, Xu Y. Concentration-dependent isoflurane effects on depolarization-evoked glutamate and GABA outflows from mouse brain slices. Br J Pharmacol. 1999;127(1):131-138.

49. McDougall SJ, Peters JH, LaBrant L, Wang X, Koop DR, Andresen MC. Paired assessment of volatile anesthetic concentrations with synaptic actions recorded in vitro. PLOS ONE. 2008;3(10):e3372.

50. Simon W, Hapfelmeier G, Kochs E, Zieglgänsberger W, Rammes G. Isoflurane blocks synaptic plasticity in the mouse hippocampus. Anesthesiology. 2001;94(6):1058-1065.

51. Farthing D, Gehr L, Berger B, Fakhry I, Sica D. Simple method for simultaneous determination of halothane, enflurane, and isoflurane in Krebs solution using capillary gas chromatography. Biomed Chromatogr. 1997;11(1):29-32.

52. Sirois JE, Pancrazio JJ, Lynch C, Bayliss DA. Multiple ionic mechanisms mediate inhibition of rat motoneurones by inhalation anaesthetics. JPhysiol (Lond). 1998;512 (Pt 3):851-862.

53. Behne M, Wilke HJ, Harder S. Clinical pharmacokinetics of sevoflurane. Clin Pharmacokinet. 1999;36(1):13-26. 